\title{
Two-level preconditioning for $h$-version boundary element approximation of hypersingular operator with GenEO
}

\author{
Pierre Marchand* Xavier Claeys* ${ }^{*}$ Pierre Jolivet ${ }^{\dagger} \quad$ Frédéric Nataf* $^{*}$ \\ Pierre-Henri Tournier*
}

July 18, 2019

\begin{abstract}
In the present contribution, we consider symmetric positive definite operators stemming from boundary integral equation (BIE), and we study a two-level preconditioner where the coarse space is built using local generalized eigenproblems in the overlap. We will refer to this coarse space as the GenEO coarse space.
\end{abstract}

Keywords - boundary integral equation, domain decomposition methods, preconditioner

\section{Introduction}

The boundary element method (BEM) is widely used to solve partial differential equations (PDE) reformulated as non-local boundary integral equations (BIE). It consists in approximating solutions of BIE using Galerkin method similarly to the finite element method (FEM). This discretization leads to linear systems

$$
\mathbf{A}_{h} \mathbf{u}_{h}=\mathbf{b}_{h},
$$

where $h$ is the mesh size of the underlying discretization. Contrary to FEM though, the non-local nature of the integral operators at play in BIE yields dense linear systems.

To solve these linear systems, one could use either a direct or iterative method. But since they are dense, compression is most of the time required, using for example fast multipole [12], hierarchical matrices [14] or sparse cardinal sine decomposition [3]. These techniques have the advantage of lowering the storage cost and make it easy to apply the operator to a vector, but the underlying structure is not well suited for direct solvers such as exact $L U$ decomposition. Nevertheless, direct solvers to be used in conjunction with a compression procedure have been developed, for example, hierarchical $L U$ decomposition [14] and a fast direct solver as described in $[21,11]$. Another approach relies on iterative solvers, such as the conjugate gradient (CG) [15] for symmetric positive definite matrices or the generalized minimal residual (GMRes) [27]. These methods have the advantage of relying only on matrix-vector products, they are non-intrusive in the sense that they are independent of the compression method used. However, the number of iterations needed strongly depends on the spectral properties of the matrix of the system. In the case of the single layer or the hypersingular operator for the Laplace problem, the condition

\footnotetext{
${ }^{*}$ Sorbonne Université, Université Paris-Diderot SPC, CNRS, Inria, Laboratoire Jacques-Louis Lions, équipe Alpines, F-75005 Paris

${ }^{\dagger}$ CNRS, Institut de Recherche en Informatique de Toulouse, 31062 Toulouse
} 
number $\kappa\left(\mathbf{A}_{h}\right):=\left\|\mathbf{A}_{h}\right\|_{2}\left\|\mathbf{A}_{h}^{-1}\right\|_{2}$ typically deteriorates like $O\left(h^{-1}\right)[28$, Section 4.5]. This means that the number of iterations when using CG will increase when refining the mesh size as the square root of $h^{-1}$.

To circumvent this problem, one has to find a relevant preconditioner, i.e., an operator $\mathbf{P}$ such that $\mathbf{P A} \mathbf{A}_{h}$ has better spectral properties (typically, a condition number bounded independently of the mesh size) and that is not too costly to apply $\mathbf{P}$ to a vector, because instead of solving the previous linear system, we will solve

$$
\mathbf{P A}_{h} \mathbf{u}_{h}=\mathbf{P b}_{h}
$$

Various preconditioning strategies have been proposed for BEM matrices. One of the most popular is Calderón preconditioning [30], which is the application of operator preconditioning [17] based on Calderón identities that shows that certain products of boundary integral operators yield a compact perturbation of the identity. Another approach consists in building a sparse approximation of the inverse to precondition our problem, which is usually called sparse approximate inverse preconditioner (SPAI) [2].

Finally, another approach, proposed in $[16,33]$ for example, relies on adapting well-known preconditioners stemming from domain decomposition methods (DDM) to BEM matrices. In the present paper, we focus on this last type of preconditioner. We made this choice based on the fact that DDM preconditioners are naturally parallel, which makes them interesting for high performance computing (HPC). Moreover, they have been extensively studied in a finite element context (see $[8,32])$.

These DDM preconditioners usually rely on a geometric decomposition of the computational domain and consists in solving local problems associated to each subdomain, but also solving a global problem of small size to add a minimal global communication between the subdomains. This global problem usually consists in solving the problem on a coarser mesh (see [32, Chapter 3 ] for example). The purpose of the global problem is to make the iterative solver scalable (see Section 4).

At the theoretical level, scalability of DDM preconditioners without a coarse component is usually not provable (see [32, Section 3.4] for a more in-depth discussion in a FEM context). The condition number of the preconditioned matrix is not bounded independently of the number of subdomains and in practice the number of iterations to solve the associated linear system increases with the number of subdomains (see [8, Chapter 4] for numerical evidences in FEM). That is why iterative solvers usually do not scale with DDM preconditioners without a coarse component.

Adding a coarse component to the preconditioner based on a coarse problem can ensure a bounded condition number, and thus scalability. It has been studied in FEM [32, Chapter 3] and this approach has been adapted to BEM [16, 33]. Recently, another kind of coarse component based on local eigenproblems has been introduced in a FEM context [29] and proved to be numerically efficient [19], namely the generalized eigenproblems in the overlap (GenEO) coarse space. It has the advantage to be more algebraic in the sense that it does not depend on the discretization or the underlying equation, as long as the latter is symmetric positive definite. In this contribution, we will introduce in the BEM context several coarse spaces inspired by GenEO. We will conduct the analysis for the hypersingular operator for symmetric positive definite problems. We will prove that such an approach ensures scalability, and we will study their efficiency numerically. 


\section{Boundary Integral Equation}

In this section, we briefly introduce the mathematical framework for boundary element methods (BEM). For more details, we refer to [22], which focuses on the functional analysis behind BIE and to [28], which presents appropriate discretization strategies and their mathematical foundations.

\subsection{Function spaces and norms}

Let $\Omega \subset \mathbb{R}^{d}$ be a Lipschitz polyhedron, and $d$ the geometric dimension, $L^{2}(\Omega)$ denotes the space of all measurable functions $u: \Omega \rightarrow \mathbb{R}$ such that $\|u\|_{L^{2}(\Omega)}^{2}:=\int_{\Omega}|u|^{2} \mathrm{~d} \mathbf{x}<\infty$. We define the fractional order Sobolev space $H^{1 / 2}(\Omega)$ as the space containing all the functions $u \in L^{2}(\Omega)$ with $\|u\|_{H^{1 / 2}(\Omega)}<\infty$, where

$$
\|\varphi\|_{H^{1 / 2}(\Omega)}^{2}:=\|\varphi\|_{L^{2}(\Omega)}^{2}+\int_{\Omega \times \Omega} \frac{|\varphi(\mathbf{x})-\varphi(\mathbf{y})|^{2}}{|\mathbf{x}-\mathbf{y}|^{d+1}} \mathrm{~d} \mathbf{x} \mathrm{d} \mathbf{y} .
$$

The second term in the last expression is usually called Slobodeckij seminorm. Now, we want to define Sobolev spaces on $\widetilde{\Gamma}:=\partial \Omega$, i.e., on curves for $d=2$ and on surfaces for $d=3$ without boundary. As we assumed $\Omega$ Lipschitz, there exists local orthogonal coordinates that represent locally $\widetilde{\Gamma}$ as the graph of a Lipschitz function of a domain of dimension $d-1$. Then, we can define $H^{1 / 2}(\widetilde{\Gamma})$ in terms of Sobolev spaces on $\mathbb{R}^{d-1}$ using surface integrals instead of volume integrals. Let $\Gamma \subseteq \widetilde{\Gamma}$ so that $\Gamma$ is a curve for $d=2$ and a surface for $d=3$ with or without boundary, we define

$$
H^{1 / 2}(\Gamma):=\left\{\left.\varphi\right|_{\Gamma} \text { s.t. } \varphi \in H^{1 / 2}(\widetilde{\Gamma})\right\} \quad \text { and } \quad \widetilde{H}^{1 / 2}(\Gamma):=\left\{\psi \in H^{1 / 2}(\Gamma) \text { s.t. } E_{\Gamma}(\psi) \in H^{1 / 2}(\widetilde{\Gamma})\right\}
$$

where $E_{\Gamma}$ is the operator of extension by zero associated with functions defined on $\Gamma$. In other words,

- $H^{1 / 2}(\Gamma)$ contains the restrictions of $H^{1 / 2}(\widetilde{\Gamma})$ to $\Gamma$,

- $\widetilde{H}^{1 / 2}(\Gamma)$ contains the functions whose extension by zero are in $H^{1 / 2}(\widetilde{\Gamma})$.

We define their associated norms as follows

$$
\begin{aligned}
\|\varphi\|_{H^{1 / 2}(\Gamma)}^{2} & :=\|\varphi\|_{L^{2}(\Gamma)}^{2}+\int_{\Gamma \times \Gamma} \frac{|\varphi(\mathbf{x})-\varphi(\mathbf{y})|^{2}}{|\mathbf{x}-\mathbf{y}|^{d+1}} \mathrm{~d} \gamma(\mathbf{x}, \mathbf{y}) \\
\|\varphi\|_{\widetilde{H}^{1 / 2}(\Gamma)} & :=\left\|E_{\Gamma}(\varphi)\right\|_{H^{1 / 2}(\widetilde{\Gamma})}
\end{aligned}
$$

where $\gamma(\mathbf{x}, \mathbf{y})$ refers to the surface measure induced on $\Gamma$ by the Lebesgue measure.

Remark. In the case $\Gamma=\widetilde{\Gamma}$, which means that $\Gamma$ does not have any boundary, we have $\widetilde{H}^{1 / 2}(\Gamma)=$ $H^{1 / 2}(\Gamma)$ with equivalent norms [22].

We also define $\widetilde{H}^{-1 / 2}(\Gamma)$ and $H^{-1 / 2}(\Gamma)$ as, respectively, the dual of $H^{1 / 2}(\Gamma)$ and $\widetilde{H}^{1 / 2}(\Gamma)$, $\langle\cdot, \cdot\rangle$ denoting the duality pairing. We endow them with the following dual norms

$$
\begin{aligned}
& \|u\|_{\widetilde{H}^{-1 / 2}(\Gamma)}:=\sup _{v \in H^{1 / 2}(\Gamma)} \frac{\langle u, v\rangle}{\|v\|_{H^{1 / 2}(\Gamma)}} \quad \forall u \in \widetilde{H}^{-1 / 2}(\Gamma), \\
& \|u\|_{H^{-1 / 2}(\Gamma)}:=\sup _{v \in \widetilde{H}^{1 / 2}(\Gamma)} \frac{\langle u, v\rangle}{\|v\|_{\widetilde{H}^{1 / 2}(\Gamma)}} \quad \forall u \in H^{-1 / 2}(\Gamma) .
\end{aligned}
$$




\subsection{BEM operator}

As a model problem, we simply consider the hypersingular integral equation: find $u \in \widetilde{H}^{1 / 2}(\Gamma)$ such that

$$
a(u, v):=\langle W u, v\rangle_{H^{-1 / 2}(\Gamma) \times \widetilde{H}^{1 / 2}(\Gamma)}=\langle f, v\rangle_{\Gamma}, \quad \forall v \in \widetilde{H}^{1 / 2}(\Gamma),
$$

where $f \in H^{-1 / 2}(\Gamma)$. The operator $W: \widetilde{H}^{1 / 2}(\Gamma) \rightarrow H^{-1 / 2}(\Gamma)$ is the hypersingular integral operator which, in the case of a Laplace problem on a screen with $d=3$, is given by

$$
\langle W u, v\rangle_{H^{-1 / 2}(\Gamma) \times \widetilde{H}^{1 / 2}(\Gamma)}=\int_{\Gamma \times \Gamma} \frac{\operatorname{curl}_{\Gamma} u(\mathbf{x}) \cdot \operatorname{curl}_{\Gamma} v(\mathbf{y})}{4 \pi|\mathbf{x}-\mathbf{y}|} \mathrm{d} \gamma(\mathbf{x}, \mathbf{y})
$$

where $\operatorname{curl}_{\Gamma} u(\mathbf{x})=\mathbf{n}(\mathbf{x}) \times \nabla_{\Gamma} u(\mathbf{x})$ and $\mathbf{n}(\mathbf{x})$ refers to the unit vector field normal to $\Gamma$ directed toward the exterior of $\Omega$. The bilinear form $a(\cdot, \cdot)$ is symmetric, continuous, and positive definite according to [31] or [28, Theorem 3.5.9.]. In the following, we can allow for a more generic symmetric bilinear form that induces a norm on $\widetilde{H}^{1 / 2}(\Gamma)$ so that, for every $u \in \widetilde{H}^{1 / 2}(\Gamma)$

$$
C^{-}\|u\|_{\widetilde{H}^{1 / 2}(\Gamma)}^{2} \leqslant a(u, u) \leqslant C^{+}\|u\|_{\widetilde{H}^{1 / 2}(\Gamma)}^{2}
$$

where $C^{-}$and $C^{+}$are positive constants.

\subsection{Discretization}

To define a discrete subspace $\mathcal{V}_{h}$, we first need to introduce a conforming triangulation of the geometric domain $\Gamma$. Let us denote $\mathcal{T}$, a mesh of $\Gamma$ and $h_{\mathcal{T}} \in L^{\infty}(\mathcal{T})$ defined by $\left.h_{\mathcal{T}}\right|_{T}:=|T|^{1 /(d-1)}$ for every $T \in \mathcal{T}$ and $h=\max _{T \in \mathcal{T}} h_{T}$. We denote $\mathcal{S}_{h}^{l}(\mathcal{T})$ the space of piecewise polynomial functions of degree $l$ on $\mathcal{T}$ and $\widetilde{\mathcal{S}}_{h}^{l}(\mathcal{T}):=\mathcal{S}_{h}^{l}(\mathcal{T}) \cap \widetilde{H}^{1 / 2}(\Gamma)$.

To discretize Equation (1), we use a standard Galerkin approximation, i.e., we solve the following problem: find $u_{h} \in \mathcal{V}_{h} \subset \widetilde{H}^{1 / 2}(\Gamma)$ such that

$$
a\left(u_{h}, v_{h}\right):=\left\langle W u_{h}, v_{h}\right\rangle_{\Gamma}=\left\langle f, v_{h}\right\rangle_{\Gamma}, \quad \forall v_{h} \in \mathcal{V}_{h},
$$

where $\mathcal{V}_{h}$ is a finite dimensional subspace of $\widetilde{H}^{1 / 2}(\Gamma)$. Let $\left(\varphi_{j}\right)_{j=1}^{N}$ be a finite element basis of $\mathcal{V}_{h}$ and $\operatorname{dim}\left(\mathcal{V}_{h}\right)=N$, so that $u_{h}=\sum_{j=1}^{N} u_{h, j} \varphi_{j}$. Then from Equation (3), we obtain the following linear system

$$
\mathbf{A}_{h} \mathbf{u}_{h}=\mathbf{b}_{h},
$$

where $\mathbf{A}_{h} \in \mathbb{R}^{N \times N}, \mathbf{b}_{h} \in \mathbb{R}^{N}$ such that $\left(\mathbf{A}_{h}\right)_{i, j}=a\left(\varphi_{j}, \varphi_{i}\right),\left(\mathbf{b}_{h}\right)_{j}=\left\langle f, \varphi_{j}\right\rangle$ for $1 \leqslant i, j \leqslant N$ and $\mathbf{u}_{h} \in \mathbb{R}^{N}$ is the vector of coefficients corresponding to the unknown finite element function $u_{h}$, so that $\left(\mathbf{u}_{h}\right)_{j}=u_{h, j}$. The matrix $\mathbf{A}_{h}$ inherits symmetric positive definiteness from $a(\cdot, \cdot)$.

\section{Domain Decomposition Methods}

We are investigating domain decomposition methods (DDM) to precondition the linear system defined in Equation (4). More precisely, we are interested in Schwarz methods, which are techniques that were first introduced as iterative procedures to approximate solutions of partial differential equations (PDE) (see [9] for a historical presentation of these methods). Afterwards, they were reinterpreted as fixed point methods with a specific preconditioner that depends on the chosen methods. That is why Schwarz methods can also refer to a preconditioner, and can be used in more efficient iterative methods, such as CG and GMRes. 


\section{$2.1 \quad$ Notations}

Let us now introduce the notations required for a proper presentation of Schwarz preconditioners. First, we need to partition the domain $\Gamma$. Since we have in mind implementation of $\mathcal{H}$-matrices, we first consider a partition of the degrees of freedom in $n$ subdomains that will be done with a geometric clustering, which induces a partition of the global numbering $\{1, \ldots, N\}=\cup_{p=1}^{n} \operatorname{dof}_{h, p}^{\prime}$ with $\operatorname{dof}_{h, p}^{\prime} \cap \operatorname{dof}_{h, l}^{\prime}=\emptyset$ for every $p, l \in\{1, \ldots, n\}$ and $p \neq l$.

This differs from what is usually done in a finite element context, where automatic partitioners such as METIS [20] are used to decompose the domain. By contrast, here we take account of a compression method working on degrees of freedom. Then, we can add several layers of mesh elements and their associated degrees of freedom to increase the overlap between neighboring subdomains. We denote the indices of the resulting $p$ th subdomain $\operatorname{dof}_{h, p}$ so that $\operatorname{dof}_{h, p}^{\prime} \subset \operatorname{dof}_{h, p}$ and $\{1, \ldots, N\} \subset \cup_{p=1}^{n} \operatorname{dof}_{h, p}$. Its associated local finite element space is

$$
\mathcal{V}_{h, p}:=\operatorname{Span}\left(\left.\varphi_{j}\right|_{\widetilde{\Gamma}_{p}} \mid j \in \operatorname{dof}_{h, p}\right) \subset \widetilde{H}^{1 / 2}\left(\widetilde{\Gamma}_{p}\right),
$$

where $\widetilde{\Gamma}_{p}:=\cup_{j \in \operatorname{dof}_{h, p}} \operatorname{supp}\left(\varphi_{\mathrm{j}}\right)$, and thus $\Gamma \subset \cup_{p=1}^{n} \widetilde{\Gamma}_{p}$. Later, we will also need to use $\Gamma_{p}:=$ $\widetilde{\Gamma}_{p} \backslash \cup_{j \notin \operatorname{dof}_{h, p}} \operatorname{supp}\left(\varphi_{\mathrm{j}}\right)$, see the remark below for an example. We define an arbitrary local numbering of the degrees of freedom with the bijection $\sigma_{p}:\left\{1,2, \ldots, N_{p}\right\} \rightarrow$ dof $_{h, p}$, where $N_{p}:=\operatorname{dim}\left(\mathcal{V}_{h, p}\right)$. Then, we can define the extension by zero at the matrix level with $\mathbf{R}_{p}^{T} \in \mathbb{R}^{N \times N_{p}}$

$$
\left(\mathbf{R}_{p}^{T}\right)_{j, k}= \begin{cases}1 & \text { if } j=\sigma_{p}(k) \\ 0 & \text { otherwise }\end{cases}
$$

and its counterpart at the function level, as

$$
\begin{gathered}
R_{p}^{T}: \mathcal{V}_{h, p} \rightarrow \mathcal{V}_{h} . \\
R_{p}^{T}\left(u_{h}^{p}\right)(\mathbf{x})=\left\{\begin{aligned}
u_{h}^{p}(\mathbf{x}) & \text { if } \mathbf{x} \in \widetilde{\Gamma}_{p} \\
0 & \text { otherwise }
\end{aligned}\right.
\end{gathered}
$$

We also define the restriction matrix $\mathbf{R}_{p} \in \mathbb{R}^{N_{p} \times N}$, as the transpose of $\mathbf{R}_{p}^{T}$ for the canonical scalar product $(\cdot, \cdot)$ on $\mathbb{R}^{N}$ and its counterpart for finite element functions

$$
\begin{aligned}
R_{p}: \mathcal{V}_{h} & \rightarrow \mathcal{V}_{h, p} . \\
\sum_{j=1}^{N} u_{h, j} \varphi_{j} & \left.\mapsto \sum_{k=1}^{N_{p}} u_{h, \sigma_{p}(k)} \varphi_{\sigma_{p}(k)}\right|_{\widetilde{\Gamma}_{p}} .
\end{aligned}
$$

Note that $R_{p}$ is not a restriction in the natural sense. It restricts the set of shape functions used to represent an element of the discrete space $\mathcal{V}_{h, p}$ (see remark thereafter). Notice also that $R_{p}^{T}$ is not the dual of $R_{p}$ for the $L^{2}$ scalar product, they are respectively the equivalent of $\mathbf{R}_{p}^{T}$ and $\mathbf{R}_{p}$ for finite element functions, and $\mathbf{R}_{p}^{T}$ is the transpose of $\mathbf{R}_{p}$.

Remark. Let us take an example and consider the case where $\Gamma$ is the interval between 0 and 10 on the $x$-axis discretized using $\mathbb{P}_{1}$ Lagrange elements $\mathcal{V}_{h}=\operatorname{Span}\left(\varphi_{j} \mid 0 \leqslant j \leqslant 10\right)$. Defining $\operatorname{dof}_{h, 1}:=\{0,1,2,3,4,5,6\}$ and $\operatorname{dof}_{h, 2}:=\{4,5,6,7,8,9,10\}$, we represent $u_{h}=1$ in Figure 1. Notice that $R_{1}\left(u_{h}\right)$ is not the usual restriction of $u_{h}$ since it is linear between 6 and 7 .

Another example in 2D is given Figure 2 for a screen with $\mathbb{P}_{1}$ shape function. The nodes in $\operatorname{dof}_{h, 1}$ are represented with rectangle nodes. We are considering a discretization of $\widetilde{H}^{1 / 2}(\Gamma)$ so that we do not have any $\mathbb{P}_{1}$ functions associated with the nodes on the boundary of the screen. 


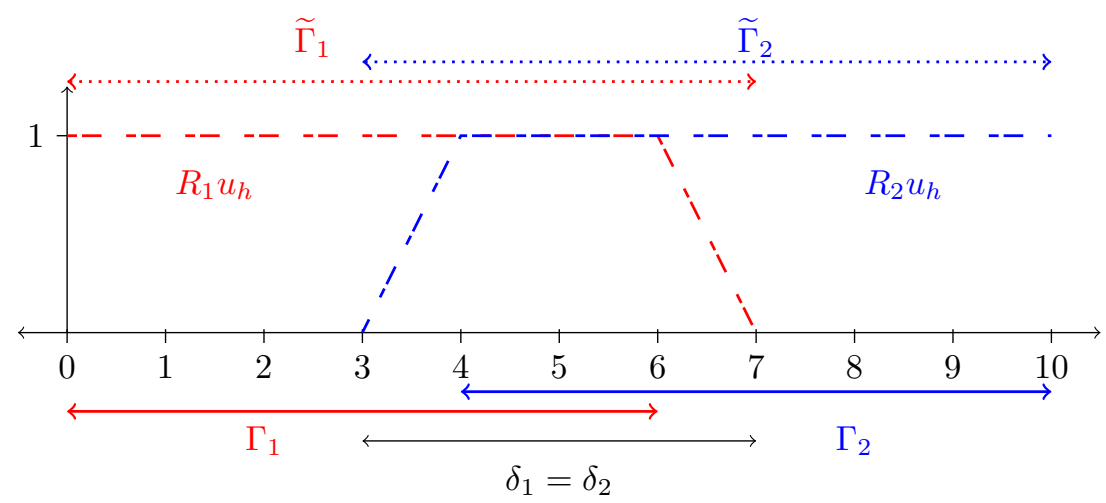

Figure 1: Example of an overlapping decomposition in 1D for $P_{1}$ finite elements with $\Gamma=\Gamma_{1} \cup \Gamma_{2}$ and $u_{h} \in \mathcal{V}_{h}$ such that $u_{h}=1$, that is to say $u_{h, j}=1$ for $1 \leqslant j \leqslant N$.
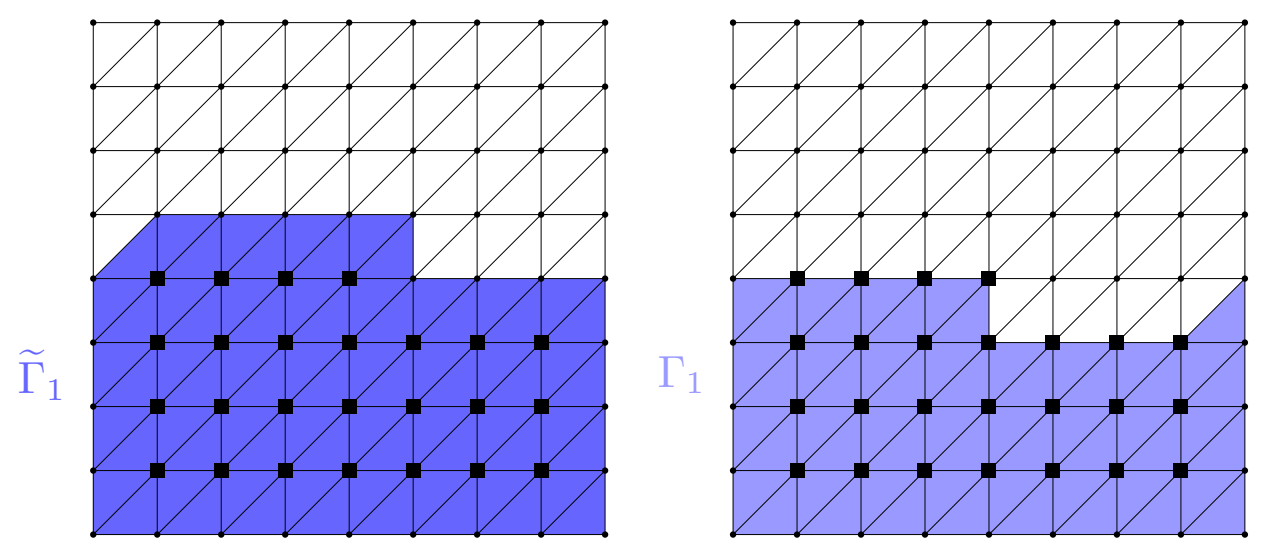

Figure 2: Example of a subdomain in 2D for $P_{1}$ finite elements where the rectangle nodes denote $P_{1}$ functions in $\mathcal{V}_{h, 1}$. 


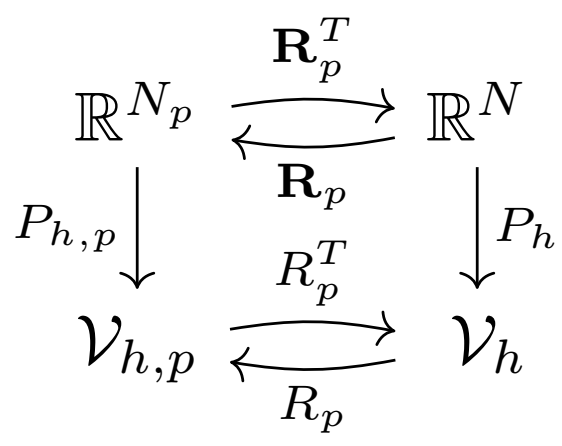

Figure 3: Relations between restriction, extension operators, $P_{h}$ and $P_{h, p}$

We have the relations $\mathbf{R}_{p} \mathbf{R}_{p}^{T}=\mathbf{I}_{d} \in \mathbb{R}^{N_{p} \times N_{p}}$ and $R_{p} R_{p}^{T}=I_{d}$. We also need to define the following linear applications

$$
\left\{\begin{array} { c } 
{ P _ { h } : \mathbb { R } ^ { N } \rightarrow \mathcal { V } _ { h } , } \\
{ \mathbf { u } _ { h } = ( u _ { h , j } ) _ { j = 1 } ^ { N } \mapsto u _ { h } = \sum _ { j = 1 } ^ { N } u _ { h , j } \varphi _ { j } , }
\end{array} \quad \text { and } \quad \left\{\begin{array}{c}
P_{h, p}: \mathbb{R}^{N_{p}} \rightarrow \mathcal{V}_{h, p}, \\
\mathbf{u}_{h}^{p}=\left(u_{h, j}^{p}\right)_{j=1}^{N_{p}} \mapsto u_{h}^{p}=\sum_{j=1}^{N_{p}} u_{h, j}^{p} \varphi_{\sigma_{p}(j)} .
\end{array}\right.\right.
$$

They allow with their inverse to go back and forth between the finite element and the algebraic point of view. Several relations exist between the operators introduced so far, they are summarized in Figure 3. Notice that we also have $\left(\mathbf{A}_{h} \mathbf{u}_{h}, \mathbf{u}_{h}\right)=a\left(P_{h} \mathbf{u}_{h}, P_{h} \mathbf{u}_{h}\right)$ with $\mathbf{u}_{h} \in \mathbb{R}^{N}$. Finally, we introduce a partition of unity $\left(\mathbf{D}_{p}\right)_{p=1}^{n} \in \prod_{p=1}^{n} \mathbb{R}^{N_{p} \times N_{p}}$ such that

$$
\mathbf{I}_{d}=\sum_{p=1}^{n} \mathbf{R}_{p}^{T} \mathbf{D}_{p} \mathbf{R}_{p} .
$$

A few geometric constants will allow us to describe the domain decomposition, and they will appear in the study of the efficiency of Schwarz preconditioners. All these definitions are inspired by [8]. We denote $k_{0}$ the maximum multiplicity of the interaction between subdomains plus one, i.e.

$$
k_{0}:=\max _{1 \leqslant j \leqslant N} \#\left\{p \mid \mathbf{R}_{p} \mathbf{M}_{h} \mathbf{R}_{j}^{T} \neq 0\right\}
$$

where $\left(\mathbf{M}_{h}\right)_{i, j}=\int_{\Gamma} \varphi_{i} \varphi_{j} \mathrm{~d} \gamma$ is the mass matrix. The maximal multiplicity of the subdomain intersection $k_{1}$ is defined as the largest integer $m$ such that there exist $m$ different subdomains whose intersection has a nonzero measure,

$$
k_{1}:=\operatorname{ess} \sup \left(\sum_{p=1}^{n} 1_{\Gamma_{p}}\right) .
$$

And finally, $N_{c}$ is the minimum number of colors we can use to color a decomposition such that any two domains sharing an overlap have different colors.

As for the domain decomposition, these constants are not supposed to increase with the number of subdomains a priori because they only depend on the local interaction between subdomains. The domain decomposition can be considered as a graph where subdomains are the 
vertices and where two vertices are related by an edge if the corresponding subdomains overlap. Using the terminology from graph theory to describe this graph, $k_{0}-1$ corresponds to the maximum vertex degree [34, Definition 1.3.1.], $N_{c}$ corresponds to the chromatic number [34, Definition 5.1.1.], and we have $N_{c} \leqslant k_{0}$ [34, Proposition 5.1.13.]. Brook's theorem [34, Theorem 5.1.22.] states that this bound can be improved to $N_{c} \leqslant k_{0}-1$ if the graph is connected [34, Definition 1.2.6.] (meaning that there is always a path between two vertices) but not complete (meaning that each vertex is not connected to every other vertex) and not an odd cycle.

\subsection{Additive Schwarz Method and coarse space}

In the present paper, we are interested in the additive schwarz method (ASM) introduced in [35] with a generic coarse space $\mathcal{V}_{h, 0} \subset \mathcal{V}_{h}$ that we will define later. We denote $N_{0}:=\operatorname{dim}\left(\mathcal{V}_{h, 0}\right)$ and it is spanned by the finite element functions associated with the columns of the rectangular matrix $\mathbf{R}_{0}^{T} \in \mathbb{R}^{N \times N_{0}}$, so that the definition of the preconditioner is

$$
\mathbf{P}_{\mathrm{ASM}}:=\mathbf{R}_{0}^{T}\left(\mathbf{R}_{0} \mathbf{A}_{h} \mathbf{R}_{0}^{T}\right)^{-1} \mathbf{R}_{0}+\sum_{p=1}^{n} \mathbf{R}_{p}^{T}\left(\mathbf{R}_{p} \mathbf{A}_{h} \mathbf{R}_{p}^{T}\right)^{-1} \mathbf{R}_{p} .
$$

Removing the coarse correction (i.e., the first term) from the above preconditioner would a priori spoil scalability. We shall discuss this in more details in Section 4.

In order to study the efficiency of the preconditioner defined in Equation (7) to bound the condition number of the preconditioned linear system associated with Equation (4), we use the fictitious space lemma $[24,13]$ or $[8$, Lemma 7.4$]$, which writes as follows when directly applied to our case.

Theorem 1. Let us denote $H_{D}:=\prod_{p=0}^{n} \mathbb{R}^{N_{p}}$, which corresponds to the space in which we decompose finite element vectors according to our domain decomposition. We endow it with the Euclidean scalar product $(\cdot, \cdot)_{D}$ for product spaces defined as $\left(u_{D}, v_{D}\right)_{D}:=\sum_{p=0}^{n}\left(\mathbf{R}_{p}^{T} \mathbf{u}^{p}, \mathbf{R}_{p}^{T} \mathbf{v}^{p}\right)$ for every $u_{D}=\left(\mathbf{u}^{p}\right)_{p=0}^{n}, v_{D}=\left(\mathbf{v}^{p}\right)_{p=0}^{n} \in H_{D}$. We also define

$$
\begin{aligned}
B: H_{D} & \rightarrow H_{D} \\
\left(\mathbf{u}_{h}^{p}\right)_{p=0}^{n} & \mapsto\left(\mathbf{R}_{p} \mathbf{A}_{h} \mathbf{R}_{p}^{T} \mathbf{u}_{h}^{p}\right)_{p=0}^{n},
\end{aligned}
$$

and

$$
\begin{aligned}
\mathcal{R}_{\mathrm{ASM}}: H_{D} & \rightarrow \mathbb{R}^{N} \\
\left(\mathbf{u}_{h}^{p}\right)_{p=0}^{n} & \mapsto \sum_{p=0}^{n} \mathbf{R}_{p}^{T} \mathbf{u}_{h}^{p} .
\end{aligned}
$$

Suppose we have the following hypotheses:

1. There exists a constant $c_{R}>0$ such that, for all $\mathbf{U}_{h}=\left(\mathbf{u}_{h}^{p}\right)_{p=0}^{n} \in H_{D}$

$$
\left(\mathbf{A}_{h} \mathcal{R}_{\mathrm{ASM}}\left(\mathbf{U}_{h}\right), \mathcal{R}_{\mathrm{ASM}}\left(\mathbf{U}_{h}\right)\right) \leqslant c_{R}\left(B \mathbf{U}_{h}, \mathbf{U}_{h}\right)_{D} .
$$

2. There exists a constant $c_{T}>0$ such that, for all $\mathbf{u}_{h} \in \mathbb{R}^{N}$, there exists $\mathbf{U}_{h} \in H_{D}$ with $\mathbf{u}_{h}=\mathcal{R}_{\mathrm{ASM}}\left(\mathbf{U}_{h}\right)$, and

$$
c_{T}\left(B \mathbf{U}_{h}, \mathbf{U}_{h}\right)_{D} \leqslant\left(\mathbf{A}_{h} \mathbf{u}_{h}, \mathbf{u}_{h}\right) .
$$


We define the adjoint operator $\mathcal{R}_{\mathrm{ASM}}^{*}: \mathbb{R}^{N} \rightarrow H_{D}$ by $\left(\mathcal{R}_{\mathrm{ASM}}\left(\mathbf{U}_{h}\right), \mathbf{u}_{h}\right)=\left(\mathbf{U}_{h}, \mathcal{R}_{\mathrm{ASM}}^{*}\left(\mathbf{u}_{h}\right)\right)_{D}$ for all $\mathbf{u}_{h} \in \mathbb{R}^{N}$ and $\mathbf{U}_{h} \in H_{D}$. Then, we have the spectral estimate

$$
\kappa\left(\mathcal{R}_{\mathrm{ASM}} B^{-1} \mathcal{R}_{\mathrm{ASM}}^{*} \mathbf{A}_{h}\right) \leqslant \frac{c_{R}}{c_{T}} .
$$

We aim at applying Theorem 1 observing that $\mathcal{R}_{\mathrm{ASM}} B^{-1} \mathcal{R}_{\mathrm{ASM}}^{*}=\mathbf{P}_{\mathrm{ASM}}$.

\section{Two-level preconditioning}

Before we introduce our two-level preconditioner, we formulate some general remarks. The first hypothesis of Theorem 1 corresponds to the continuity of $\mathcal{R}_{\mathrm{ASM}}$ and the second one is usually referred to as stable decomposition. Using the notations of this theorem, the two hypotheses can be rewritten as follows

1. There exists a constant $c_{R}>0$ such that, for all $\left(\mathbf{u}_{h}^{p}\right)_{p=0}^{n} \in H_{D}$

$$
a\left(\sum_{p=0}^{n} P_{h} \mathbf{R}_{p}^{T} \mathbf{u}_{h}^{p}, \sum_{p=0}^{n} P_{h} \mathbf{R}_{p}^{T} \mathbf{u}_{h}^{p}\right) \leqslant c_{R} \sum_{p=0}^{n} a\left(P_{h} \mathbf{R}_{p}^{T} \mathbf{u}_{h}^{p}, P_{h} \mathbf{R}_{p}^{T} \mathbf{u}_{h}^{p}\right) .
$$

2. There exists a constant $c_{T}>0$ such that, for all $\mathbf{u}_{h} \in \mathbb{R}^{N}$, there exists $\mathbf{U}_{h}=\left(\mathbf{u}_{h}^{p}\right)_{p=0}^{n} \in H_{D}$ with $\mathbf{u}_{h}=\mathcal{R}_{\mathrm{ASM}}\left(\mathbf{U}_{h}\right)$, and

$$
c_{T} \sum_{p=0}^{n} a\left(P_{h} \mathbf{R}_{p}^{T} \mathbf{u}_{h}^{p}, P_{h} \mathbf{R}_{p}^{T} \mathbf{u}_{h}^{p}\right) \leqslant a\left(P_{h} \mathbf{u}_{h}, P_{h} \mathbf{u}_{h}\right) .
$$

We deduce that these two hypotheses are related to how we can "localize" the bilinear form $a$, and since it is symmetric positive definite, it is also related to how we can localize the $\widetilde{H}^{1 / 2}$-norm.

\subsection{Continuity of $\mathcal{R}_{\mathrm{ASM}}$}

The first hypothesis of Theorem 1 is actually satisfied without any precise definition of the coarse space. We have the following theorem to localize the $\widetilde{H}^{1 / 2}$-norm

Lemma 1 ([28, Lemma 4.1.49 (b)]). For $\left(u_{p}\right)_{1 \leqslant p \leqslant n} \in \prod_{p=1}^{n} \widetilde{H}^{1 / 2}\left(\Gamma_{p}^{\prime}\right)$ with $\left(\Gamma_{p}^{\prime}\right)_{p=1}^{n}$ a nonoverlapping partition of $\Gamma$, i.e. $\Gamma=\cup_{p=1}^{n} \Gamma_{p}^{\prime}$ and $\Gamma_{p}^{\prime} \cap \Gamma_{l}^{\prime}=\emptyset$ for $l \neq p$ and $1 \leqslant l, p \leqslant n$, we have the following inequality:

$$
\left\|\sum_{p=1}^{n} E_{\Gamma_{p}^{\prime}}\left(u_{p}\right)\right\|_{\widetilde{H}^{1 / 2}(\Gamma)}^{2} \leqslant \frac{5}{2} \sum_{p=1}^{n}\left\|u_{p}\right\|_{\widetilde{H}^{1 / 2}\left(\Gamma_{p}^{\prime}\right)}^{2},
$$

Then, we can obtain the following lemma using a coloring argument:

Lemma 2. For $\left(u_{p}\right)_{1 \leqslant p \leqslant n} \in \prod_{p=1}^{n} \widetilde{H}^{1 / 2}\left(\widetilde{\Gamma}_{p}\right)$, we have the following inequality:

$$
\left\|\sum_{p=1}^{n} E_{\widetilde{\Gamma}_{p}}\left(u_{p}\right)\right\|_{\widetilde{H}^{1 / 2}(\Gamma)}^{2} \leqslant \frac{5}{2} N_{c} \sum_{p=1}^{n}\left\|u_{p}\right\|_{\widetilde{H}^{1 / 2}\left(\widetilde{\Gamma}_{p}\right)}^{2} .
$$


Proof. If one colors each subdomain $\widetilde{\Gamma}_{p}$ such that two subdomains with the same color cannot be neighbors, that is to say, cannot overlap, then we can define $\left(\Gamma_{l}^{\prime}\right)_{l=1}^{N_{c}}$ where each $\Gamma_{l}^{\prime}$ is the union of every subdomain with the same color, that we number $l$, and $N_{c}$ is the number of colors. Let us define

$$
w_{l}=\sum_{p \mid \widetilde{\Gamma}_{p} \subset \Gamma_{l}^{\prime}} E_{\widetilde{\Gamma}_{p}}\left(u_{p}\right) \in \widetilde{H}^{1 / 2}\left(\Gamma_{l}^{\prime}\right)
$$

Then, we have

$$
\left\|\sum_{p=1}^{n} E_{\widetilde{\Gamma}_{p}}\left(u_{p}\right)\right\|_{\widetilde{H}^{1 / 2}(\Gamma)}^{2}=\left\|\sum_{l=1}^{N_{c}} \sum_{p \mid \widetilde{\Gamma}_{p} \subset \Gamma_{l}^{\prime}} E_{\widetilde{\Gamma}_{p}}\left(u_{p}\right)\right\|_{\widetilde{H}^{1 / 2}(\Gamma)}^{2}=\left\|\sum_{l=1}^{N_{c}} w_{l}\right\|_{\widetilde{H}^{1 / 2}(\Gamma)}^{2} \leqslant N_{c} \sum_{l=1}^{N_{c}}\left\|w_{l}\right\|_{\widetilde{H}^{1 / 2}(\Gamma)}^{2} .
$$

By definition of $\Gamma_{l}^{\prime}$, we have that all subdomains $\widetilde{\Gamma}_{p}$ such that $\widetilde{\Gamma}_{p} \subset \Gamma_{l}^{\prime}$ are disjoint so that we can use Lemma 1 in the last expression to obtain

$$
\begin{aligned}
& N_{c} \sum_{l=1}^{N_{c}}\left\|w_{l}\right\|_{\widetilde{H}^{1 / 2}(\Gamma)}^{2} \leqslant N_{c} \sum_{l=1}^{N_{c}}\left\|\sum_{p \mid \widetilde{\Gamma}_{p} \subset \Gamma_{l}^{\prime}} E_{\widetilde{\Gamma}_{p}}\left(u_{p}\right)\right\|_{\widetilde{H}^{1 / 2}(\Gamma)}^{2} \\
& \leqslant N_{c} \frac{5}{2} \sum_{l=1}^{N_{c}} \sum_{p \mid \widetilde{\Gamma}_{p} \subset \Gamma_{l}^{\prime}}\left\|u_{p}\right\|_{\widetilde{H}^{1 / 2}}^{2}\left(\widetilde{\Gamma}_{p}\right)=N_{c} \frac{5}{2} \sum_{p=1}^{n}\left\|u_{p}\right\|_{\widetilde{H}^{1 / 2}\left(\widetilde{\Gamma}_{p}\right)}^{2} .
\end{aligned}
$$

The last lemma yields an upper bound for the global energy on $\widetilde{H}^{1 / 2}(\Gamma)$ by a sum of local energies, so that we obtain the first hypothesis required by Theorem 1 .

Lemma 3. Using the notations of Theorem 1, let $\mathbf{U}_{h}=\left(\mathbf{u}_{h}^{p}\right)_{p=1}^{n} \in H_{D}$, we have

$$
\left(\mathbf{A}_{h} \mathcal{R}_{\mathrm{ASM}}\left(\mathbf{U}_{h}\right), \mathcal{R}_{\mathrm{ASM}}\left(\mathbf{U}_{h}\right)\right) \leqslant 2 \max \left(1, \frac{5}{2} N_{c} \frac{C^{+}}{C_{-}}\right)\left(B \mathbf{U}_{h}, \mathbf{U}_{h}\right)_{D} .
$$

Proof. Using the fact that $\mathbf{A}_{h}$ is symmetric positive definite, we have

$$
\begin{aligned}
\left(\mathbf{A}_{h} \mathcal{R}_{\mathrm{ASM}}\left(\mathbf{U}_{h}\right),\right. & \left.\mathcal{R}_{\mathrm{ASM}}\left(\mathbf{U}_{h}\right)\right)=\left(\mathbf{A}_{h} \sum_{p=0}^{n} \mathbf{R}_{p}^{T} \mathbf{u}_{h}^{p}, \sum_{p=0}^{n} \mathbf{R}_{p}^{T} \mathbf{u}_{h}^{p}\right) \\
& \leqslant 2\left(\left(\mathbf{A}_{h} \mathbf{R}_{0}^{T} \mathbf{u}_{h}^{0}, \mathbf{R}_{0}^{T} \mathbf{u}_{h}^{0}\right)+\left(\mathbf{A}_{h} \sum_{p=1}^{n} \mathbf{R}_{p}^{T} \mathbf{u}_{h}^{p}, \sum_{p=1}^{n} \mathbf{R}_{p}^{T} \mathbf{u}_{h}^{p}\right)\right) .
\end{aligned}
$$

Notice that the last term on the right-hand side can be rewritten

$$
\begin{aligned}
\left(\mathbf{A}_{h} \sum_{p=1}^{n} \mathbf{R}_{p}^{T} \mathbf{u}_{h}^{p}, \sum_{p=1}^{n} \mathbf{R}_{p}^{T} \mathbf{u}_{h}^{p}\right) & =a\left(\sum_{p=1}^{n} P_{h} \mathbf{R}_{p}^{T} \mathbf{u}_{h}^{p}, \sum_{p=1}^{n} P_{h} \mathbf{R}_{p}^{T} \mathbf{u}_{h}^{p}\right) \\
& =a\left(\sum_{p=1}^{n} R_{p}^{T} u_{h}^{p}, \sum_{p=1}^{n} R_{p}^{T} u_{h}^{p}\right)
\end{aligned}
$$


with $u_{h}^{p}=P_{h, p} \mathbf{u}_{h}^{p} \in \mathcal{V}_{h, p} \subset \widetilde{H}^{1 / 2}\left(\widetilde{\Gamma}_{p}\right)$ (see diagram Figure 3). Finally, using the equivalence relation from Equation (2), Lemma 2 and the definition of the $\widetilde{H}^{1 / 2}$-norm, we have

$$
\begin{aligned}
a\left(\sum_{p=1}^{n} R_{p}^{T} u_{h}^{p}, \sum_{p=1}^{n} R_{p}^{T} u_{h}^{p}\right) & \leqslant C^{+}\left\|\sum_{p=1}^{n} R_{p}^{T} u_{h}^{p}\right\|_{\widetilde{H}^{1 / 2}(\Gamma)}^{2} \leqslant \frac{5}{2} N_{c} C^{+} \sum_{p=1}^{n}\left\|u_{h}^{p}\right\|_{\widetilde{H}^{s}\left(\widetilde{\Gamma}_{p}\right)}^{2} \\
& \leqslant \frac{5}{2} N_{c} C^{+} \sum_{p=1}^{n}\left\|R_{p}^{T} u_{h}^{p}\right\|_{\widetilde{H}^{s}(\Gamma)}^{2} \leqslant \frac{5}{2} N_{c} \frac{C^{+}}{C_{-}} \sum_{p=1}^{n} a\left(R_{p}^{T} u_{h}^{p}, R_{p}^{T} u_{h}^{p}\right) \\
& \leqslant \frac{5}{2} N_{c} \frac{C^{+}}{C_{-}} \sum_{p=1}^{n}\left(\mathbf{A}_{h} \mathbf{R}_{p}^{T} \mathbf{u}_{h}^{p}, \mathbf{R}_{p}^{T} \mathbf{u}_{h}^{p}\right) .
\end{aligned}
$$

We would like to point out that, although one can obtain similar results to Lemma 2 with other norms (with interpolation for example), we use in the last lemma the fact that $\left\|u_{h}^{p}\right\|_{\widetilde{H}^{1 / 2}\left(\Gamma_{p}\right)}=$ $\left\|R_{p}^{T} u_{h}^{p}\right\|_{\widetilde{H}^{1 / 2}(\Gamma)}$ which is not a priori guaranteed for other norms. And even if an equivalence relation can be proved, extra care must be taken to show that the constants in the equivalence relation are independent of the size of the subdomain $\Gamma_{p}$ which is related to the number of subdomains. As a consequence, the choice of the norm $\|\cdot\|_{\widetilde{H}^{1 / 2}(\Gamma)}$ plays a key role in the present analysis.

\subsection{Stable decomposition - GenEO concept}

We now focus on the second hypothesis of Theorem 1 . The goal is to find a way to decompose global finite element functions so that we can bound the global energy from below by the sum of the local energies. To do so, let us explain the idea behind the GenEO approach. First, using the results from the preceding section, we have the following lemma for a generic coarse space.

Lemma 4. Using the notations of Theorem 1 , let $\mathbf{u}_{h} \in \mathbb{R}^{N}$ and $\mathbf{U}_{h}=\left(\mathbf{u}_{h}^{p}\right)_{p=0}^{n} \in \prod_{p=0}^{n} \mathbb{R}^{N_{p}}$ such that $\mathbf{u}_{h}=\mathcal{R}_{\mathrm{ASM}}\left(\mathbf{U}_{h}\right)$. Then, we have

$$
\left(B \mathbf{U}_{h}, \mathbf{U}_{h}\right)_{D} \leqslant 2\left(\mathbf{A}_{h} \mathbf{u}_{h}, \mathbf{u}_{h}\right)+\left(1+5 N_{c} \frac{C^{+}}{C^{-}}\right) \sum_{p=1}^{n}\left(\mathbf{A}_{h} \mathbf{R}_{p}^{T} \mathbf{u}_{h}^{p}, \mathbf{R}_{p}^{T} \mathbf{u}_{h}^{p}\right) .
$$

Proof. Using the definition of $B$ and the Cauchy-Schwarz inequality,

$$
\begin{aligned}
\left(B \mathbf{U}_{h}, \mathbf{U}_{h}\right)_{D} & =\left(\mathbf{A}_{h} \mathbf{R}_{0}^{T} \mathbf{u}_{h}^{0}, \mathbf{R}_{0}^{T} \mathbf{u}_{h}^{0}\right)+\sum_{p=1}^{n}\left(\mathbf{A}_{h} \mathbf{R}_{p}^{T} \mathbf{u}_{h}^{p}, \mathbf{R}_{p}^{T} \mathbf{u}_{h}^{p}\right) \\
& =\left(\mathbf{A}_{h}\left(\mathbf{u}_{h}-\sum_{p=1}^{n} \mathbf{R}_{p}^{T} \mathbf{u}_{h}^{p}\right), \mathbf{u}_{h}-\sum_{p=1}^{n} \mathbf{R}_{p}^{T} \mathbf{u}_{h}^{p}\right)+\sum_{p=1}^{n}\left(\mathbf{A}_{h} \mathbf{R}_{p}^{T} \mathbf{u}_{h}^{p}, \mathbf{R}_{p}^{T} \mathbf{u}_{h}^{p}\right) \\
& \leqslant 2\left(\left(\mathbf{A}_{h} \mathbf{u}_{h}, \mathbf{u}_{h}\right)+\left(\mathbf{A}_{h} \sum_{p=1}^{n} \mathbf{R}_{p}^{T} \mathbf{u}_{h}^{p}, \sum_{p=1}^{n} \mathbf{R}_{p}^{T} \mathbf{u}_{h}^{p}\right)\right)+\sum_{p=1}^{n}\left(\mathbf{A}_{h} \mathbf{R}_{p}^{T} \mathbf{u}_{h}^{p}, \mathbf{R}_{p}^{T} \mathbf{u}_{h}^{p}\right) .
\end{aligned}
$$

Then, we apply the equivalence relation from Equation (2) and Lemma 2 to the second term in the right-hand side to obtain the desired result. 
The last lemma is insufficient to prove a spectral estimate of the ASM preconditioner. There remains to bound the last term, corresponding to the sum of local energies, by the global energy $a\left(P_{h} \mathbf{u}_{h}, P_{h} \mathbf{u}_{h}\right)$. Let us assume there exists $\left(\mathbf{B}_{p}\right)_{p=1}^{n} \in \prod_{p=1}^{n} \mathbb{R}^{N_{p} \times N_{p}}$ and a constant $C_{\text {loc }}>0$ independent of $h$ and $n$ such that

$$
C_{\text {loc }} \sum_{p=1}^{n}\left(\mathbf{B}_{p} \mathbf{R}_{p} \mathbf{u}_{h}, \mathbf{R}_{p} \mathbf{u}_{h}\right) \leqslant a\left(P_{h} \mathbf{u}_{h}, P_{h} \mathbf{u}_{h}\right) .
$$

To obtain the second hypothesis of Theorem 1, and using Lemma 4 with Equation (8), one can see that a sufficient condition would be to find a decomposition $\mathbf{U}_{h}=\left(\mathbf{u}_{h}^{p}\right)_{p=0}^{n} \in \prod_{p=0}^{n} \mathbb{R}^{N_{p}}$ of a given $\mathbf{u}_{h} \in \mathbb{R}^{N}$ such that

$$
\left(\mathbf{A}_{h} \mathbf{R}_{p}^{T} \mathbf{u}_{h}^{p}, \mathbf{R}_{p}^{T} \mathbf{u}_{h}^{p}\right) \leqslant \tau\left(\mathbf{B}_{p} \mathbf{R}_{p} \mathbf{u}_{h}, \mathbf{R}_{p} \mathbf{u}_{h}\right)
$$

for some fixed user-defined parameter $\tau>0$ and $\mathbf{u}_{h}=\mathcal{R}_{\mathrm{ASM}}\left(\mathbf{U}_{h}\right)$. A natural choice for $\left(\mathbf{u}_{h}^{p}\right)_{p=0}^{n}$ might be $\mathbf{u}_{h}^{p}=\mathbf{D}_{p} \mathbf{R}_{p} \mathbf{u}_{h}$, but generally it does not satisfy Equation (9). The idea of the GenEO coarse space is to filter out the part of $\mathbf{D}_{p} \mathbf{R}_{p} \mathbf{u}_{h}$ that does not satisfy Equation (9) using the following local generalized eigenvalue problem: find $\left(\mathbf{v}_{h, k}^{p}, \lambda_{k}^{p}\right)$ such that

$$
\mathbf{D}_{p} \mathbf{R}_{p} \mathbf{A}_{h} \mathbf{R}_{p}^{T} \mathbf{D}_{p} \mathbf{v}_{h, k}^{p}=\lambda_{k}^{p} \mathbf{B}_{p} \mathbf{v}_{h, k}^{p} .
$$

Then, we can define the local contribution to the coarse space

$$
Z_{p, \tau}:=\operatorname{ker}\left(\mathbf{B}_{p}\right) \cup \operatorname{Span}\left(\mathbf{v}_{h, k}^{p} \mid \text { for every } k \text { s.t. } \lambda_{k}^{p}>\tau\right),
$$

and the local projection $\pi_{p}$ on $Z_{p, \tau}$ parallel to $\operatorname{Span}\left(\mathbf{v}_{h, k}^{p} \mid\right.$ for every $k$ s.t. $\left.\lambda_{k}^{p} \leqslant \tau\right)$. Using the projection $\pi_{p}$, we can filter out the part of $\mathbf{D}_{p} \mathbf{R}_{p} \mathbf{u}_{h}$ that does not satisfy the sufficient condition given by Equation (9).

Lemma 5 ([8, Lemma 7.15]). For every $1 \leqslant p \leqslant n$ and $\mathbf{v}_{h, k} \in \mathbb{R}^{N_{p}}$, we have

$$
\left(\mathbf{R}_{p}^{T} \mathbf{D}_{p}\left(\mathbf{I}_{d}-\pi_{p}\right) \mathbf{v}_{h}^{p}\right)^{T} \mathbf{A}_{h}\left(\mathbf{R}_{p}^{T} \mathbf{D}_{p}\left(\mathbf{I}_{d}-\pi_{p}\right) \mathbf{v}_{h}^{p}\right) \leqslant \tau\left(\mathbf{v}_{h}^{p}\right)^{T} \mathbf{B}_{p} \mathbf{v}_{h}^{p} .
$$

The last relation is similar to Equation (9) so that we define the decomposition such that, for $1 \leqslant p \leqslant n$

$$
\mathbf{u}_{h}^{p}=\mathbf{D}_{p}\left(\mathbf{I}_{d}-\pi_{p}\right) \mathbf{R}_{p} \mathbf{u}_{h} .
$$

It remains to define the coarse space $\mathcal{V}_{h, 0}$ and the associated coarse component $\mathbf{u}_{h}^{0}$ such that $\mathbf{u}_{h}=\mathcal{R}_{\mathrm{ASM}, 2}\left(\mathbf{U}_{h}\right)$ with $\mathbf{U}_{h}=\left(\mathbf{u}_{h}^{p}\right)_{p=0}^{n}$.

Definition 1. The GenEO coarse space is defined as the sum of the local contributions to the coarse space weighted with the partition of unity. We define

$$
\mathcal{V}_{h, 0}=\operatorname{Span}\left(\mathbf{R}_{p}^{T} \mathbf{D}_{p} \mathbf{v}_{h}^{p} \mid 1 \leq p \leq N, \mathbf{v}_{h}^{p} \in Z_{p, \tau}\right)
$$

Let $\mathbf{Z}_{\tau} \in \mathbb{R}^{N \times N_{0}}$ be a column matrix so that $\mathcal{V}_{h, 0}$ is spanned by its columns and $N_{0}=\operatorname{dim}\left(\mathcal{V}_{h, 0}\right)$. We denote its transpose by $\mathbf{R}_{0}:=\mathbf{Z}_{\tau}^{T}$.

Lemma 6 (GenEO coarse component). Assuming there exists $\left(\mathbf{B}_{p}\right)_{p=1}^{n}$ such that Equation (8) is true, we can define the coarse space as in Definition 1, and the coarse component as

$$
\mathbf{u}_{h}^{0}:=\left(\mathbf{R}_{0} \mathbf{R}_{0}^{T}\right)^{-1} \mathbf{R}_{0}\left(\sum_{p=1}^{n} \mathbf{R}_{p}^{T} \mathbf{D}_{p} \pi_{p} \mathbf{R}_{p} \mathbf{u}_{h}\right)
$$


then $\mathbf{U}_{h}=\left(\mathbf{u}_{h}^{p}\right)_{p=0}^{n} \in H_{D}$ defined by Equations (12) and (13) is a stable decomposition of $\mathbf{u}_{h}$, that is to say

$$
\mathcal{R}_{\mathrm{ASM}, 2}\left(\mathbf{U}_{h}\right)=\mathbf{u}_{h} \quad \text { and } \quad c_{T} \sum_{p=0}^{n} a\left(P_{h} \mathbf{R}_{p}^{T} \mathbf{u}_{h}^{p}, P_{h} \mathbf{R}_{p}^{T} \mathbf{u}_{h}^{p}\right) \leqslant a\left(P_{h} \mathbf{u}_{h}, P_{h} \mathbf{u}_{h}\right),
$$

with $c_{T}^{-1}=2+\left(1+5 N_{c} \frac{C^{+}}{C^{-}}\right) \frac{\tau}{C_{\mathrm{loc}}}$.

Proof. Let $\mathbf{u}_{h} \in \mathbb{R}^{N}$ and $\mathbf{U}_{h}=\left(\mathbf{u}_{h}^{p}\right)_{0 \leqslant p \leqslant n} \in H_{D}$ be defined by Equations (12) and (13). First notice that we have

$$
\mathbf{w}_{0}=\mathbf{R}_{0}^{T}\left(\mathbf{R}_{0} \mathbf{R}_{0}^{T}\right)^{-1} \mathbf{R}_{0} \mathbf{w}_{0}
$$

for every $\mathbf{w}_{0} \in \mathcal{V}_{h, 0}$ since $\mathbf{R}_{0}^{T}\left(\mathbf{R}_{0} \mathbf{R}_{0}^{T}\right)^{-1} \mathbf{R}_{0}$ is a projector on $\mathcal{V}_{h, 0}$. Then, we have

$$
\begin{aligned}
\mathcal{R}_{\mathrm{ASM}, 2}\left(\mathbf{U}_{h}\right) & =\sum_{p=0}^{n} \mathbf{R}_{p}^{T} \mathbf{u}_{h}^{p}=\mathbf{R}_{0}^{T} \mathbf{u}_{h}^{0}+\sum_{p=1}^{n} \mathbf{R}_{p}^{T} \mathbf{u}_{h}^{p} \\
& =\mathbf{R}_{0}^{T}\left(\mathbf{R}_{0} \mathbf{R}_{0}^{T}\right)^{-1} \mathbf{R}_{0} \underbrace{\left(\sum_{p=1}^{n} \mathbf{R}_{p}^{T} \mathbf{D}_{p} \pi_{p} \mathbf{R}_{p} \mathbf{u}_{h}\right)}_{\in \mathcal{V}_{h, 0}}+\sum_{p=1}^{n} \mathbf{R}_{p}^{T} \mathbf{u}_{h}^{p} \\
& =\sum_{p=1}^{n} \mathbf{R}_{p}^{T} \mathbf{D}_{p} \pi_{p} \mathbf{R}_{p} \mathbf{u}_{h}+\sum_{p=1}^{n} \mathbf{R}_{p}^{T} \mathbf{D}_{p}\left(\mathbf{I}_{d}-\pi_{p}\right) \mathbf{R}_{p} \mathbf{u}_{h} \\
& =\sum_{p=1}^{n} \mathbf{R}_{p}^{T} \mathbf{D}_{p} \mathbf{R}_{p} \mathbf{u}_{h}=\mathbf{u}_{h},
\end{aligned}
$$

where we used the fact that $\mathbf{D}_{p}$ defines a partition of unity in the last line. Then, using Lemmas 4 and 5 with $\mathbf{v}_{h}^{p}=\mathbf{R}_{p} \mathbf{u}_{h}$ for $1 \leqslant p \leqslant n$, we have

$$
\begin{aligned}
\sum_{p=0}^{n} a\left(P_{h} \mathbf{R}_{p}^{T} \mathbf{u}_{h}^{p}, P_{h} \mathbf{R}_{p}^{T} \mathbf{u}_{h}^{p}\right) & =\sum_{p=0}^{n}\left(\mathbf{A}_{h} \mathbf{R}_{p}^{T} \mathbf{u}_{h}^{p}, \mathbf{R}_{p}^{T} \mathbf{u}_{h}^{p}\right) \\
& \leqslant 2\left(\mathbf{A}_{h} \mathbf{u}_{h}, \mathbf{u}_{h}\right)+\left(1+5 N_{c} \frac{C^{+}}{C^{-}}\right) \sum_{p=1}^{n}\left(\mathbf{A}_{h} \mathbf{R}_{p}^{T} \mathbf{u}_{h}^{p}, \mathbf{R}_{p}^{T} \mathbf{u}_{h}^{p}\right) \\
& \leqslant 2\left(\mathbf{A}_{h} \mathbf{u}_{h}, \mathbf{u}_{h}\right)+\left(1+5 N_{c} \frac{C^{+}}{C^{-}}\right) \tau \sum_{p=1}^{n}\left(\mathbf{B}_{p} \mathbf{R}_{p} \mathbf{u}_{h}, \mathbf{R}_{p} \mathbf{u}_{h}\right) .
\end{aligned}
$$

Finally, assuming Equation (8) holds, we deduce

$$
\sum_{p=0}^{n} a\left(P_{h} \mathbf{R}_{p}^{T} \mathbf{u}_{h}^{p}, P_{h} \mathbf{R}_{p}^{T} \mathbf{u}_{h}^{p}\right) \leqslant\left(2+\left(1+5 N_{c} \frac{C^{+}}{C^{-}}\right) \frac{\tau}{C_{\mathrm{loc}}}\right) a\left(P_{h} \mathbf{u}_{h}, P_{h} \mathbf{u}_{h}\right) .
$$

Remark. It should be noted that the decomposition $\left(\mathbf{u}_{h}^{p}\right)_{p=1}^{n}$ is necessary for the analysis, but it does not need to be computed in practice. To use this coarse space, it is sufficient to compute $\mathbf{Z}_{\tau}$ 
and then $\left(\mathbf{R}_{0} \mathbf{A}_{h} \mathbf{R}_{0}^{T}\right)^{-1}$ to be able to apply $\mathbf{P}_{A S M}$ (see definition given in Equation (7)). And to do so, each local contribution to the coarse space defined in Equation (11) can be computed independently in parallel, so that computing $\mathbf{Z}_{\tau}$ can be done efficiently. Then, to compute $\mathbf{R}_{0} \mathbf{A}_{h} \mathbf{R}_{0}^{T}$, one can use the fact that $\mathbf{Z}_{\tau}$ is sparse and distributed (since the eigenvectors are computed in parallel). Finally, we need to invert $\left(\mathbf{R}_{0} \mathbf{A}_{h} \mathbf{R}_{0}^{T}\right) \in \mathbb{R}^{N_{0} \times N_{0}}$ and that is why a good coarse space should be of minimum size while still containing relevant information for the convergence.

\subsection{Concrete coarse spaces}

Using Lemmas 3 and 6 , it remains to find a sequence of local operators $\left(\mathbf{B}_{p}\right)_{p=1}^{n}$ that satisfies Equation (8) to be able to apply Theorem 1. The goal is to find a coarse space whose size is as small as possible for a given $\tau$ because we need to invert $\mathbf{R}_{0} \mathbf{A}_{h} \mathbf{R}_{0}^{T} \in \mathbb{R}^{N_{0} \times N_{0}}$. However, the choice of the set $\left(\mathbf{B}_{p}\right)_{p=1}^{n}$ is not a priori unique, and we do not know the size of the coarse space in advance for a given $\tau$, so that numerical tests are necessary to see which one is the most efficient. A first simple way to obtain a decomposition $\left(\mathbf{B}_{p}\right)_{p=1}^{n}$ satisfying Equation (8) is to simply use the continuous injectivity between $\widetilde{H}^{1 / 2}(\Gamma)$ and $L^{2}(\Gamma)$.

Lemma 7. Let $u \in \widetilde{H}^{1 / 2}(\Gamma)$, we have

$$
\frac{C_{\mathrm{inj}} C^{-}}{k_{1}} \sum_{p=1}^{n}\|u\|_{L^{2}\left(\Gamma_{p}\right)}^{2} \leqslant a(u, u)
$$

where $k_{1}$ is defined in Equation (6), $C_{\mathrm{inj}}$ is the continuity constant of the injection of $\widetilde{H}^{1 / 2}(\Gamma)$ in $L^{2}(\Gamma)$, and they are both independent of $h$ and $n$.

Proof. Using the fact that we have $\widetilde{H}^{1 / 2}(\Gamma) \hookrightarrow L^{2}(\Gamma)$, we obtain

$$
\begin{aligned}
a(u, u) & \geqslant C^{-}\|u\|_{\widetilde{H}^{1 / 2}(\Gamma)}^{2} \geqslant C_{\mathrm{inj}} C^{-}\|u\|_{L^{2}(\Gamma)}^{2} \\
& \geqslant \frac{C_{\mathrm{inj}} C^{-}}{k_{1}} \sum_{p=1}^{n}\|u\|_{L^{2}\left(\Gamma_{p}\right)}^{2} .
\end{aligned}
$$

Using the last lemma, we obtain a similar relation to Equation (8) with $C_{\mathrm{loc}}=\frac{C_{\mathrm{inj}} C^{-}}{k_{1}}$ and $\mathbf{B}_{p}=\mathbf{M}_{p}$ for every $1 \leqslant p \leqslant n$ where $\mathbf{M}_{p}$ is the mass matrix defined as

$$
\left(\mathbf{M}_{p}\right)_{i, j}:=\int_{\Gamma_{p}} \varphi_{\sigma_{p}(i)} \varphi_{\sigma_{p}(j)} \mathrm{d} \gamma
$$

where $\Gamma_{p}$ has been defined so that $\mathbf{M}_{p} \in \mathbb{R}^{N_{p} \times N_{p}}$. Then, we can obtain the following theorem

Theorem 2. We have the following condition number estimate

$$
\kappa\left(\mathbf{P}_{\mathrm{ASM}, \text { inj }} \mathbf{A}_{h}\right) \leqslant 2 \max \left(1, N_{c} \frac{5}{2} \frac{C^{+}}{C^{-}}\right)\left(2+\left(1+5 N_{c} \frac{C^{+}}{C^{-}}\right) \frac{\tau k_{1}}{C_{\mathrm{inj}} C^{-}}\right),
$$

where $\mathbf{P}_{\mathrm{ASM}, \mathrm{inj}}$ is defined with the GenEO coarse space and the localization from Lemma $\%$.

Proof. Using Theorem 1 and its notations, we have to satisfy its two hypotheses.

1. The first hypothesis is obtained using Lemma 3 with $c_{R}=2 \max \left(1, N_{c} \frac{5 C^{+}}{2 C^{-}}\right)$. 
2. The second hypothesis is obtained using Lemma 6 with $\mathbf{B}_{p}=\mathbf{M}_{p}$ for every $1 \leqslant p \leqslant n$ and Lemma 7 so that $c_{T}^{-1}=2+\left(1+5 N_{c} \frac{C^{+} k_{1}}{C^{-}}\right) \frac{\tau k_{1}}{C_{\text {inj }} C^{-}}$.

We will refer to the coarse space based on Lemma 7 as GenEO mass coarse space. Another approach to build a coarse space is to use a well-established inequality from adaptive BEM [5, Corollary 6] to obtain the next lemma.

Lemma 8. Let $u_{h} \in \widetilde{S}^{l}\left(\mathcal{T}_{h}\right)$, we have

$$
\frac{C_{\mathrm{ad}} C^{-}}{k_{1}} \sum_{p=1}^{n}\left\|h_{\mathcal{T}}^{1 / 2} \nabla_{\Gamma} u_{h}\right\|_{L^{2}\left(\Gamma_{p}\right)}^{2} \leqslant a\left(u_{h}, u_{h}\right),
$$

where $C_{\mathrm{ad}}$ depends only on $\Gamma$, the shape-regularity of $\mathcal{T}_{h}$ and $l$.

Proof. According to [5, Corollary 6], we have

$$
\begin{aligned}
a\left(u_{h}, u_{h}\right) & \geqslant C^{-}\left\|u_{h}\right\|_{\widetilde{H}^{1 / 2}(\Gamma)}^{2} \geqslant C_{\mathrm{ad}} C^{-}\left\|h_{\mathcal{T}}^{1 / 2} \nabla_{\Gamma} u_{h}\right\|_{L^{2}(\Gamma)}^{2} \\
& \geqslant \frac{C_{\mathrm{ad}} C^{-}}{k_{1}} \sum_{p=1}^{n}\left\|h_{\mathcal{T}}^{1 / 2} \nabla u_{h}\right\|_{L^{2}\left(\Gamma_{p}\right)}^{2} .
\end{aligned}
$$

Using the last lemma, we obtain a similar relation to Equation (8) with $C_{\mathrm{loc}}=\frac{C_{\mathrm{ad}} C^{-}}{k_{1}}$ and $\mathbf{B}_{p}=\mathbf{K}_{p}$ for every $1 \leqslant p \leqslant n$ where $\mathbf{K}_{p}$ is the stiffness matrix defined as

$$
\left(\mathbf{K}_{p}\right)_{i, j}:=\int_{\Gamma_{p}} h_{\mathcal{T}} \nabla_{\Gamma} \varphi_{\sigma_{p}(i)} \nabla_{\Gamma} \varphi_{\sigma_{p}(j)} \mathrm{d} \gamma,
$$

where again, $\mathbf{K}_{p} \in \mathbb{R}^{N_{p} \times N_{p}}$ due to the definition of $\Gamma_{p}$. Then, we can obtain the following theorem

Theorem 3. We have the following condition number estimate

$$
\kappa\left(\mathbf{P}_{\mathrm{ASM}, \mathrm{ad}} \mathbf{A}_{h}\right) \leqslant 2 \max \left(1, N_{c} \frac{5}{2} \frac{C^{+}}{C^{-}}\right)\left(2+\left(1+5 N_{c} \frac{C^{+}}{C^{-}}\right) \frac{\tau k_{1}}{C_{\mathrm{ad}} C^{-}}\right),
$$

where $\mathbf{P}_{\mathrm{ASM}, \text { ad }}$ is defined with the GenEO coarse space and the localization from Lemma 8.

Proof. Using Theorem 1 and its notations, we have to satisfy its two hypotheses.

1. The first hypothesis is obtained using Lemma 3 with $c_{R}=2 \max \left(1, N_{c} \frac{5 C^{+}}{2 C^{-}}\right)$.

2. The second hypothesis is obtained using Lemma 6 with $\mathbf{B}_{p}=\mathbf{K}_{p}$ for every $1 \leqslant p \leqslant n$ and Lemma 8 so that $c_{T}^{-1}=2+\left(1+5 N_{c} \frac{C^{+} k_{1}}{C^{-}}\right) \frac{\tau k_{1}}{C_{\text {ad }} C^{-}}$.

We will refer to the coarse space based on Lemma 8 as GenEO stiffness coarse space. A third approach is to directly split the $\widetilde{H}^{1 / 2}$-norm, but we cannot directly split this norm in a sum of local contributions of $\widetilde{H}^{1 / 2}$-norms. Actually this kind of inequality has been proved wrong in the appendix of [1]. But we can bound $\widetilde{H}^{1 / 2}$-norm from below by a sum of local contributions of $H^{1 / 2}$-norms using the following lemma 
Lemma 9. For $u \in H^{1 / 2}(\Gamma)$, there holds

$$
\frac{C^{-}}{k_{1}} \sum_{p=1}^{n}\left\|\left.u\right|_{\Gamma_{p}}\right\|_{H^{1 / 2}\left(\Gamma_{p}\right)}^{2} \leqslant a(u, u),
$$

where $k_{1}$ is defined in Equation (6).

Proof. The proof is in two parts. First, we bound the $\widetilde{H}^{1 / 2}$-norm from below by the $H^{1 / 2}$-norm, and then we split the $H^{1 / 2}$-norm. We denote

$$
\int_{\Gamma} \int_{\Gamma^{\prime}}[u]:=\int_{\Gamma} \int_{\Gamma^{\prime}} \frac{|u(\mathbf{x})-u(\mathbf{y})|^{2}}{|\mathbf{x}-\mathbf{y}|^{d+1}} \mathrm{~d} \gamma(\mathbf{x}, \mathbf{y}),
$$

where we borrowed the notation from [28, Lemma 4.1 .49 (b)].

- First part. We have $\Gamma \subset \widetilde{\Gamma}=\partial \Omega$ with $\Omega \subset \mathbb{R}^{d}$, a Lipschitz polyhedron. Then, for $u \in \widetilde{H}^{1 / 2}(\Gamma)$, we have by definition

$$
\begin{aligned}
\|u\|_{\widetilde{H}^{1 / 2}(\Gamma)}^{2} & =\left\|E_{\Gamma}(u)\right\|_{H^{1 / 2}(\widetilde{\Gamma})}^{2}=\int_{\Gamma}|u|^{2}+\int_{\widetilde{\Gamma}} \int_{\widetilde{\Gamma}}\left[E_{\Gamma}(u)\right] \\
& =\underbrace{\|u\|_{L^{2}(\Gamma)}^{2}+\int_{\Gamma} \int_{\Gamma}[u]}_{=\|u\|_{H^{1 / 2}(\Gamma)}^{2}}+\underbrace{2 \int_{\widetilde{\Gamma} \backslash \Gamma} \int_{\Gamma}[u]+\int_{\widetilde{\Gamma} \backslash \Gamma} \int_{\widetilde{\Gamma} \backslash \Gamma}[u]}_{\geqslant 0} \\
& \geqslant\|u\|_{H^{1 / 2}(\Gamma)}^{2} .
\end{aligned}
$$

— Second part. According to the definition of the norm, we have

$$
\|u\|_{H^{1 / 2}(\Gamma)}^{2}=\|u\|_{L^{2}(\Gamma)}^{2}+\int_{\Gamma} \int_{\Gamma}[u] .
$$

We already used the fact that $\sum_{p=1}^{n}\left\|\left.u\right|_{\Gamma_{p}}\right\|_{L^{2}\left(\Gamma_{p}\right)}^{2} \leqslant k_{1}\|u\|_{L^{2}(\Gamma)}^{2}$ to prove Lemma 7, it remains to prove $\sum_{p=1}^{n} \int_{\Gamma_{p}} \int_{\Gamma_{p}}[u] \leqslant k_{1} \int_{\Gamma} \int_{\Gamma}[u]$.

Let $1 \leqslant l \leqslant n$, we consider all the intersections of $l$ subdomains among the $n$ subdomains. Note that there can be several areas shared by possibly different sets of $l$ subdomains, see Figure 4 for an example. That is why, we define an arbitrary numbering $\left\{1, \ldots, n_{l}\right\}$ of all the set of $l$ subdomains that intersect each other. More precisely, we define

$$
\Delta_{l}=\left\{\left\{i_{1}, \ldots, i_{l}\right\} \subset\{1, \ldots, n\} \text { s.t. } 1 \leqslant i_{1}, \ldots, i_{l} \leqslant n \text { and } \cap_{i=1}^{l} \Gamma_{i_{k}} \neq \emptyset\right\}=\left\{\Delta_{l, i}\right\}_{i=1}^{n_{l}} .
$$

Let us denote $\theta_{l, i}=\cap_{k \in \Delta_{l, i}} \Gamma_{k}$ the $i$ th part of $\Gamma$ shared by $l$ subdomains. In particular, we have $\theta_{1, p}:=\Gamma_{p} \backslash \overline{\left(\cup_{m=1, m \neq p}^{n} \Gamma_{m}\right)}$, i.e., $\theta_{1, p}$ corresponds to the $p$ th subdomain without its overlap. We denote

$$
\Theta:=\left\{(l, i) \in \mathbb{N}^{2} \mid \theta_{l, i} \neq \emptyset\right\},
$$

and remark that $\left\{\theta_{l, i}\right\}_{l, i}$ defines a partition of $\Gamma$, so that

$$
\int_{\Gamma} \int_{\Gamma}[u]=\sum_{(l, i) \in \Theta} \sum_{(m, j) \in \Theta} \int_{\theta_{l, i}} \int_{\theta_{m, j}}[u] .
$$




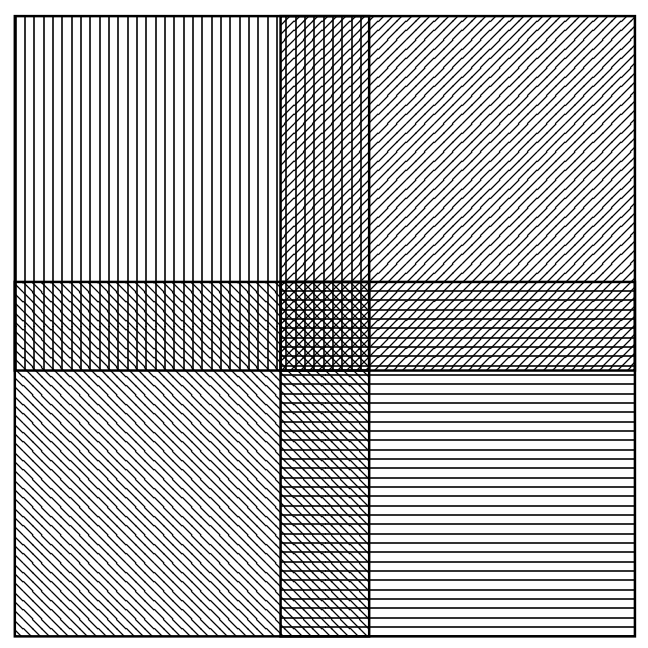

\begin{tabular}{|c|c|c|}
\hline$\theta_{1,3}$ & $\theta_{2,3}$ & $\theta_{1,4}$ \\
& & \\
\hline$\theta_{2,4}$ & $\theta_{4,1}$ & $\theta_{2,2}$ \\
\hline$\theta_{1,1}$ & $\theta_{2,1}$ & $\theta_{1,2}$ \\
\hline
\end{tabular}

Figure 4: Example of a partition defined as in the proof of Lemma 9.

For every subdomain $\Gamma_{p}$, we also define

$$
\Theta_{p}:=\left\{(l, i) \in \mathbb{N}^{2} \mid \theta_{l, i} \cap \Gamma_{p} \neq \emptyset\right\},
$$

so that $\Gamma_{p}=\cup_{(l, i) \in \Theta_{p}} \theta_{l, i}$ and

$$
\begin{aligned}
\sum_{p=1}^{n} \int_{\Gamma_{p}} \int_{\Gamma_{p}}[u] & =\sum_{p=1}^{n} \sum_{\left(l_{p}, i_{p}\right) \in \Theta_{p}} \sum_{\left(m_{p}, j_{p}\right) \in \Theta_{p}} \int_{\theta_{l_{p}, i_{p}}} \int_{\theta_{m_{p}, j_{p}}}[u] \\
& =\sum_{p=1}^{n} \sum_{(l, i) \in \Theta} \sum_{(m, j) \in \Theta} \mathbb{1}_{(l, i) \in \Theta_{p}} \mathbb{1}_{(m, j) \in \Theta_{p}} \int_{\theta_{l, i}} \int_{\theta_{m, j}}[u] \\
& =\sum_{(l, i) \in \Theta} \sum_{(m, j) \in \Theta} \sum_{p=1}^{n} \mathbb{1}_{(l, i) \in \Theta_{p}} \mathbb{1}_{(m, j) \in \Theta_{p}} \int_{\theta_{l, i}} \int_{\theta_{m, j}}[u],
\end{aligned}
$$

where $\mathbb{1}_{(l, i) \in \Theta_{p}}=1$ if $(l, i) \in \Theta_{p}$ and 0 otherwise. And, by definition, a given pair $(l, i) \in \Theta$ cannot be in more that $k_{1}$ sets $\Theta_{p}$, otherwise it would mean that $\theta_{l, i}$ is shared by more than $k_{1}$ subdomains. Thus,

$$
\sum_{p=1}^{n} \mathbb{1}_{(l, i) \in \Theta_{p}} \mathbb{1}_{(m, j) \in \Theta_{p}} \leqslant k_{1} .
$$

Finally, we obtain the expected result using Equations (16) and (17) associated with the previous inequality.

Using the last lemma, we obtain a similar relation to Equation (8) with $C_{\mathrm{loc}}=\frac{C^{-}}{k_{1}}$ and $\mathbf{B}_{p}=\mathbf{H}_{p}$ for every $1 \leqslant p \leqslant n$ where $\mathbf{H}_{p}$ is the matrix associated with the scalar product of $H^{1 / 2}(\Gamma)$ defined as

$$
\left(\mathbf{H}_{p}\right)_{i, j}:=\int_{\Gamma_{p}} \varphi_{\sigma_{p}(i)} \varphi_{\sigma_{p}(j)} \mathrm{d} \gamma+\int_{\Gamma_{p}} \int_{\Gamma_{p}} \frac{\left(\varphi_{\sigma_{p}(i)}(\mathbf{x})-\varphi_{\sigma_{p}(i)}(\mathbf{y})\right)\left(\varphi_{\sigma_{p}(j)}(\mathbf{x})-\varphi_{\sigma_{p}(j)}(\mathbf{y})\right)}{|\mathbf{x}-\mathbf{y}|^{d+1}} \mathrm{~d} \gamma(\mathbf{x}, \mathbf{y}),
$$


where again, $\mathbf{H}_{p} \in \mathbb{R}^{N_{p} \times N_{p}}$ due to the definition of $\Gamma_{p}$. Then, we can obtain the following theorem

Theorem 4. We have the following condition number estimate

$$
\kappa\left(\mathbf{P}_{\mathrm{ASM}, \mathrm{slo}} \mathbf{A}_{h}\right) \leqslant 2 \max \left(1, N_{c} \frac{5}{2} \frac{C^{+}}{C^{-}}\right)\left(2+\left(1+5 N_{c} \frac{C^{+}}{C^{-}}\right) \frac{\tau k_{1}}{C^{-}}\right),
$$

where $\mathbf{P}_{\mathrm{ASM}, \text { slo }}$ is defined with the GenEO coarse space and the localization from Lemma 9.

Proof. Using Theorem 1 and its notations, we have to satisfy its two hypotheses.

1. The first hypothesis is obtained using Lemma 3 with $c_{R}=2 \max \left(1, N_{c} \frac{5 C^{+}}{2 C^{-}}\right)$.

2. The second hypothesis is obtained using Lemma 6 with $\mathbf{B}_{p}=\mathbf{H}_{p}$ for every $1 \leqslant p \leqslant n$ and Lemma 9 so that $c_{T}^{-1}=2+\left(1+5 N_{c} \frac{C^{+} k_{1}}{C^{-}}\right) \frac{\tau k_{1}}{C^{-}}$.

We will refer to the coarse space based on Lemma 9 as GenEO Slobodeckij. Another possibility is to approximate the $H^{1 / 2}$-norm in Lemma 9. It is natural to introduce the weakly singular operator $V: \widetilde{H}^{-1 / 2}(\Gamma) \rightarrow H^{1 / 2}(\Gamma)$, whose range is the right space under consideration. We suppose that $V$ is symmetric positive definite, which is true when we assume that the global hypersingular operator $W$ also has this property. Then, we can define the local weakly singular operator $V_{p}$ as

$$
\left\langle V_{p} u_{p}, v_{p}\right\rangle_{H^{1 / 2}\left(\Gamma_{p}\right) \times \widetilde{H}^{-1 / 2}\left(\Gamma_{p}\right)}:=\left\langle V E_{\Gamma_{p}}\left(u_{p}\right), E_{\Gamma_{p}}\left(v_{p}\right)\right\rangle_{H^{1 / 2}(\Gamma) \times \widetilde{H}^{-1 / 2}(\Gamma)}
$$

for every $u_{p}, v_{p} \in \widetilde{H}^{-1 / 2}\left(\Gamma_{p}\right)$. Now, we can define a norm on $H^{1 / 2}\left(\Gamma_{p}\right)$ using the inverse of the local weakly singular operator and equivalent to $\|u\|_{H^{1 / 2}\left(\Gamma_{p}\right)}^{2}$. We are still working on its analysis, and it would be too much of a digression to present it here. That is why, we only define the associated discrete local operator $\mathbf{B}_{p}=\widetilde{\mathbf{C}}_{p}:=\mathbf{M}_{p} \mathbf{V}_{p}^{-1} \mathbf{M}_{p}$, for every $1 \leqslant p \leqslant n$ where $\mathbf{V}_{p}$ is the discretization of the single layer defined as

$$
\mathbf{V}_{p}(i, j):=\left\langle V_{p}\left(\varphi_{\sigma_{p}(j)} \mid \Gamma_{p}\right),\left.\varphi_{\sigma_{p}(i)}\right|_{\Gamma_{p}}\right\rangle_{H^{1 / 2}\left(\Gamma_{p}\right) \times \widetilde{H}^{-1 / 2}\left(\Gamma_{p}\right)},
$$

where again, $\widetilde{\mathbf{C}}_{p} \in \mathbb{R}^{N_{p} \times N_{p}}$ due to the definition of $\Gamma_{p}$. The choice of $\widetilde{\mathbf{C}}_{p}$ comes from the fact that we need to approximate the discretization $V_{p}^{-1}$ by the inverse of the discretization of $V_{p}$, which is classical in Calderón preconditioning, see $[23,30]$. We will refer to this coarse space as GenEO single layer.

In conclusion, we have introduced four coarse spaces and Theorems 2 to 4 show that at least three of them lead to a bounded condition number for the preconditioned linear systems independently of the mesh size and the number of subdomains. The size of the coarse spaces will adapt to the user-defined parameter $\tau$, which is one of the advantages of this approach. The downside, though, is that we do not know these sizes a priori. For example, if one has to take all the eigenvectors in the local generalized eigenproblem defined in Equation (10), the size of the coarse space will be the size of the global matrix, and thus inverting $\mathbf{R}_{0} \mathbf{A}_{h} \mathbf{R}_{0}^{T}$ will be as costly as using a direct method. We have to verify how relevant these coarse spaces are numerically, and which one retains the most of relevant information. In other words, we have to check numerically that the sizes of the coarse spaces are small compared to the size of the global problem, and which one is the smallest while improving the convergence. 
The only intuition we can have a priori is about GenEO single layer. Indeed, looking at the associated local generalized eigenproblem, it can be rewritten as a local eigenproblem

$$
\mathbf{M}_{p}^{-1} \mathbf{V}_{p} \mathbf{M}_{p}^{-1} \mathbf{D}_{p} \mathbf{R}_{p} \mathbf{A}_{h} \mathbf{R}_{p}^{T} \mathbf{D}_{p} \mathbf{v}_{h, k}^{p}=\lambda_{k}^{p} \mathbf{v}_{h, k}^{p}
$$

The matrix product appearing in the left hand side of Equation (20) is really close to a local Calderón preconditioning apart from $\mathbf{D}_{p}$. The subdomains usually correspond to screens and it is a well-known fact that Calderón preconditioning does not work well in this case. In [18, Fig. 4.1] for example, we can see that a few high eigenvalues appear, which is what we want in our approach.

In Figure 5, we show the spectra obtained for Equation (20) using the geometry described in Figure 6 with eight subdomains, $h=0.1$ and the problem described in Section 4.1. We see that GenEO single layer, GenEO Slobodeckij and GenEO stiffness have a few high eigenvalues so that we know at least that we should be able to discriminate a few eigenvectors for these coarse spaces. We see that we obtain the same type of spectrum for GenEO single layer as in [18]. We also observe that the spectrum obtained with the GenEO mass coarse space is not adequate with our approach, and we will see afterward that it does not contain enough relevant information to improve the convergence.

\section{Numerical experiments}

To see if the previous coarse spaces are well-adapted in practice, we need to verify that they improve scalability. Several definitions of scalability can be given. For strong scalability tests, we study how the number of iterations varies when we increase the number of subdomains for a given problem size. By contrast, for weak scalability tests the local problem size is kept constant, in other words doubling the number of subdomains means doubling the global problem size. In both cases, the method is said to be scalable if the number of iterations does not increase with the number of subdomains. In a HPC context, where we usually assign one subdomain per core, this yields robust methods in terms of computing times (for example, constant computing time for weak scalability tests). Indeed, the computing time should be proportional to the size of the subdomains and the number of iterations, provided the coarse problem $\left(\mathbf{R}_{0} \mathbf{A}_{h} \mathbf{R}_{0}^{T}\right)$ is small enough to be solved efficiently with a direct method.

According to Theorems 2 to 4, we see that the condition number of the linear system used in Equation (4), preconditioned by the additive Schwarz preconditioner defined in Equation (7) with the coarse spaces we introduced, is independent of the number or size of subdomains. The numerical experiments in this section will illustrate Theorems 2 to 4 with up to 512 subdomains and show that the proposed methods are scalable, as the resulting coarse problems remain small enough for a direct solver.

Note that the preconditioners we introduced are independent of compression techniques, but one has to implement them efficiently with a compression technique to be able to run tests of reasonable size. That is why we developed HTool, an open source $\mathrm{C}++$ library for hierarchical matrices that offers with HPDDM a way to use DDM preconditioners with MPI and OpenMP.

In particular, it allows to illustrate numerically the efficiency of the previous coarse spaces and their costs. We use BemTool ${ }^{1}$ to compute the coefficients associated with the interaction between two degrees of freedom and a $\mathbb{P}_{1}$-Lagrange discretization, $\mathrm{HTool}^{2}$ to compress the matrices using hierarchical matrices as described in [6, 14, 25] and HPDDM [19] which provides iterative solvers. The setup of the DDM preconditioners for BEM matrices is shared between HPDDM and HTool.

\footnotetext{
${ }^{1}$ https://github.com/xclaeys/BemTool

2 https://github.com/PierreMarchand20/htool
} 

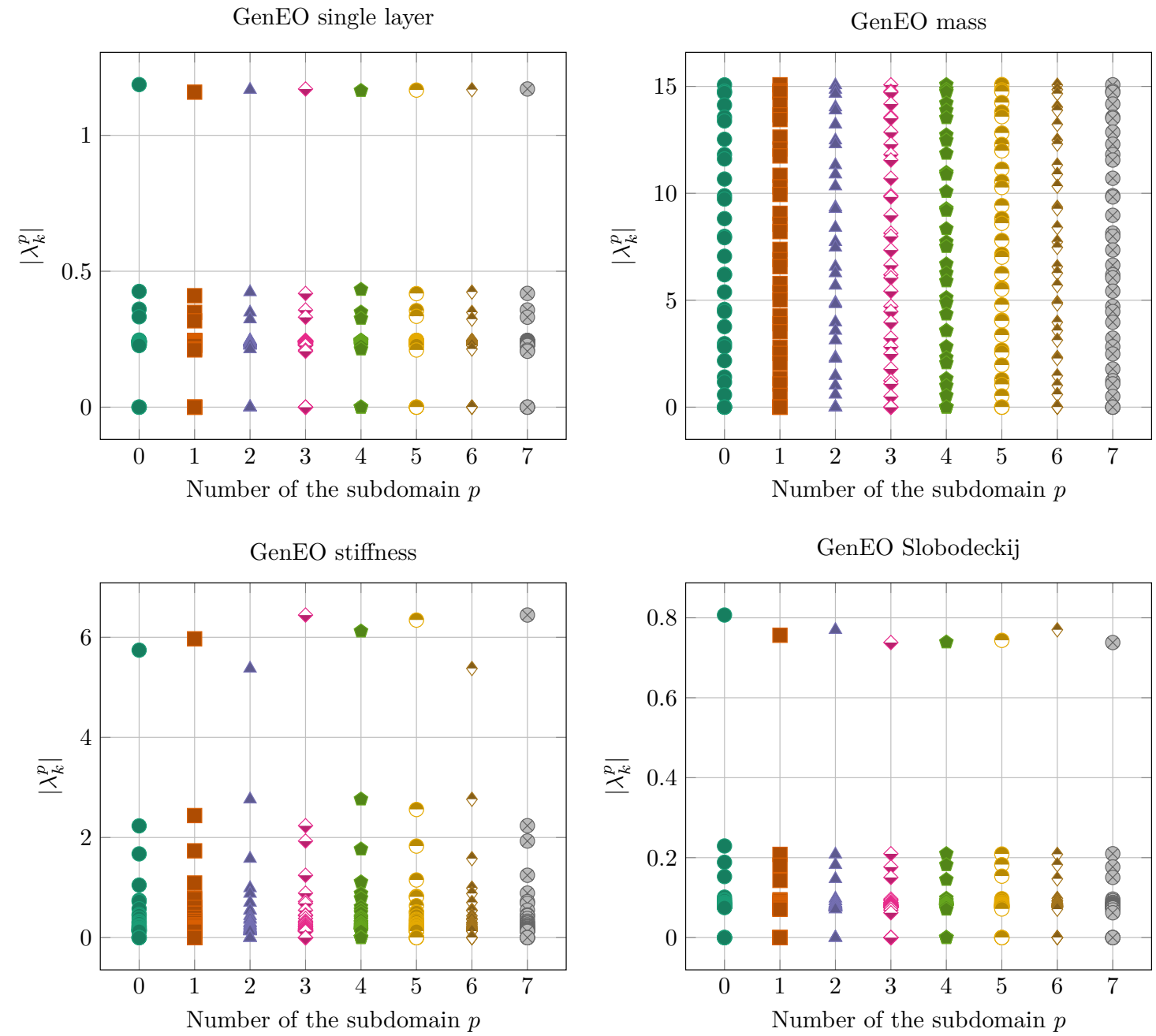

Figure 5: Spectrum obtained from Equation (20) with the geometry described in Figure 6 and eight subdomains. 
Concerning compression, hierarchical matrices are built upon a hierarchical decomposition of the degrees of freedom in clusters that is usually called cluster tree. Each block of the global matrix can be seen as the interaction between two nodes of this tree. Then, we define the block tree as the product of this cluster tree with itself, so that each node corresponds to a block in the global matrix. Because of the nature of the integral kernels, some of these blocks can be considered as admissible meaning that they correspond to far interactions so that they can be well approximated using low-rank matrices, while others are not admissible because they are associated with close interactions. In the latter case, one has to look at the subblocks via the block tree or the block needs to be built as a dense block.

To determine if a block is admissible, we use the admissibility condition described in [25, (3.15)]. A block is considered to be admissible if the minimum of the diameters of both clusters over the distance separating them is lower than a user-defined parameter $\eta$. Then, we use Adaptive Cross Approximation with partial pivoting similarly to [25, Algorithm 3.9] to compress the admissible blocks. The stopping criterion of this algorithm looks at the Frobenius norm between two consecutive approximations and if it is lower than a given threshold $\epsilon$. In our numerical tests, we take $\eta=10$ and $\epsilon=0.01$. Note that the compression may depend on how the hierarchical matrix is parallelized, but in the case of the strong scaling, we made sure that the compression was the same to have a fair comparison with different subdomains.

Our domain decomposition is actually defined by the cluster tree. More precisely, one of the levels in the cluster tree defines a partition without overlap, to which we add overlap. Notice that the algorithm used to build the cluster tree tries to do a balanced decomposition between the clusters of nodes to have a better compression and load-balanced decomposition.

We would like to point out that we are using Gmsh [10] to create all the meshes in our test cases and LAPACK [4] to solve generalized eigenproblems. Besides, we use right preconditioning, we set the tolerance for the iterative solvers in HPDDM to $10^{-6}$, and we have not used any restart with GMRes.

\subsection{D test case}

Let $\Omega_{\text {out }}=[-2,2] \times[-2,2] \subset \mathbb{R}^{2}$ and $\Omega_{\text {in }}=[-1,1] \times[-1,1] \subset \mathbb{R}^{2}$. In this first numerical experiment, we want to solve the following equation

$$
-\Delta u+\kappa^{2} u=0, \quad \text { in } \Omega:=\overline{\Omega_{\text {out }} \backslash \Omega_{\text {in }}}
$$

where $\kappa=0.1$ with the Neumann condition $\left.u\right|_{\partial \Omega}=f(x, y)$ such that

$$
f(x, y)=\left\{\begin{array}{rr}
100 \times(x+1.5)^{2} & \text { if } x>1.5 \\
0 & \text { otherwise }
\end{array}\right.
$$

This problem can be reformulated with a BIE according to [28, Section 3.4.1.1] as Equation (1) but with

$$
\begin{aligned}
\langle W u, v\rangle_{H^{-1 / 2}(\Gamma) \times \widetilde{H}^{1 / 2}(\Gamma)}= & \int_{\Gamma \times \Gamma} G(\mathbf{x}-\mathbf{y}) \operatorname{curl}_{\Gamma} u(\mathbf{x}) \cdot \operatorname{curl}_{\Gamma} v(\mathbf{y}) \mathrm{d} \gamma(\mathbf{x}, \mathbf{y}) \\
& +\kappa^{2} \int_{\Gamma \times \Gamma} G(\mathbf{x}-\mathbf{y})(\mathbf{n}(\mathbf{x}), \mathbf{n}(\mathbf{y})) u(\mathbf{x}) v(\mathbf{y}) \mathrm{d} \gamma(\mathbf{x}, \mathbf{y})
\end{aligned}
$$

with $G(\mathbf{z}):=\frac{1}{4} i H_{0}^{(1)}(i \kappa)$ where $H_{0}^{(1)}$ is the Hankel function of order zero and of first kind (see [7, $\S 10.2(\mathrm{ii})]$ ). The bilinear form is symmetric positive definite according to [28, Theorem 3.5.4]. 


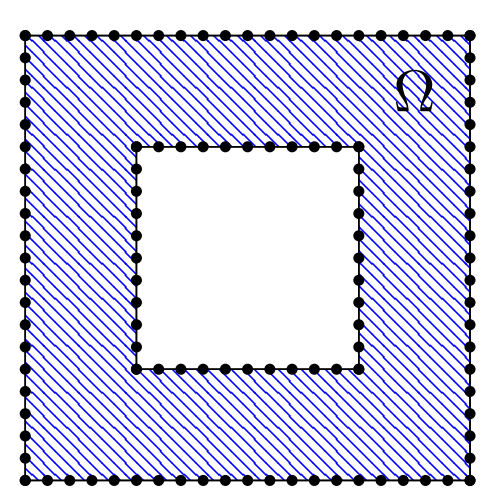

Figure 6: 2D geometry

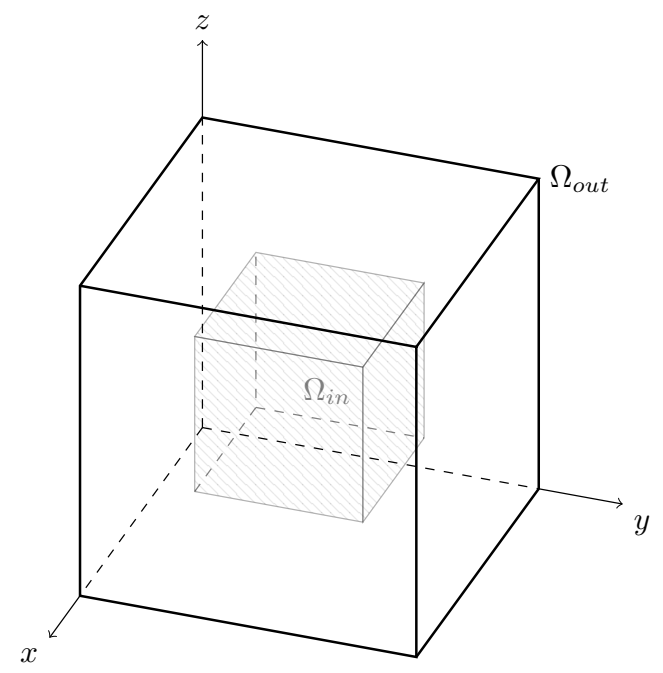

Figure 7: 3D geometry

\section{$4.2 \quad 2 \mathrm{D}$ strong scaling}

We consider a discretization of the geometry described in Figure 6 with a mesh size $h=0.001$ so that the number of degrees of freedom $N$ is equal to 24000 . We solve the linear system associated with our test case and preconditioned with the coarse spaces introduced in the previous section for several numbers of subdomains: $\{8,16,32,64,128\}$. We only add one layer of elements for the overlap. To have a fair comparison between these coarse spaces, we take $\tau=60$ for the GenEO stiffness coarse space, and we take the same number of eigenvectors in the local eigenproblems to build the coarse component of the other coarse spaces, so that the sizes of the local contributions to the coarse space and the size of the global coarse space are the same for a given number of subdomains with all the coarse spaces.

The resulting numbers of iterations using CG and GMRes are given in Figure 8. Since $\tau$ is fixed, the condition number is bounded independently of the number of subdomains according to Theorem 3 for GenEO stiffness, so that we expect the number of iterations to be constant contrary to the case without the coarse component.

The numbers of iterations without preconditioner are 656 for CG and 450 for GMRes, and they do not depend on the number of subdomains so that they are not shown in Figure 8 . We first observe that the preconditioner without coarse component greatly reduces the number of iterations since it is approximately between 40 and 120 iterations instead of several hundreds without preconditioner. But as expected, the one-level preconditioner does not scale with the number of subdomains. It goes from 57 iterations for 8 subdomains to 114 iterations for 128 subdomains with CG for example. We do not show the results for the GenEO mass coarse space because the results are at best close to the one-level. We conclude that this coarse space needs to be much larger to scale in terms of iterations. The GenEO Slobodeckij, GenEO stiffness and GenEO single layer coarse spaces scale, their number of iterations stagnates respectively between 10 and 27 iterations for CG. Note that the number of iterations for the latter is always greater than the other two. It shows that the size of the GenEO single layer coarse space needs to be greater to maintain the same amount of information. We call the number of eigenvectors taken to build $Z_{p, \tau}$, local contribution to the global coarse space associated with the $p$ th subdomain and we show Figure 9 how the mean of these local contributions and the size of the global coarse space 
Strong scaling in $2 \mathrm{D}$ with $\mathrm{CG}$

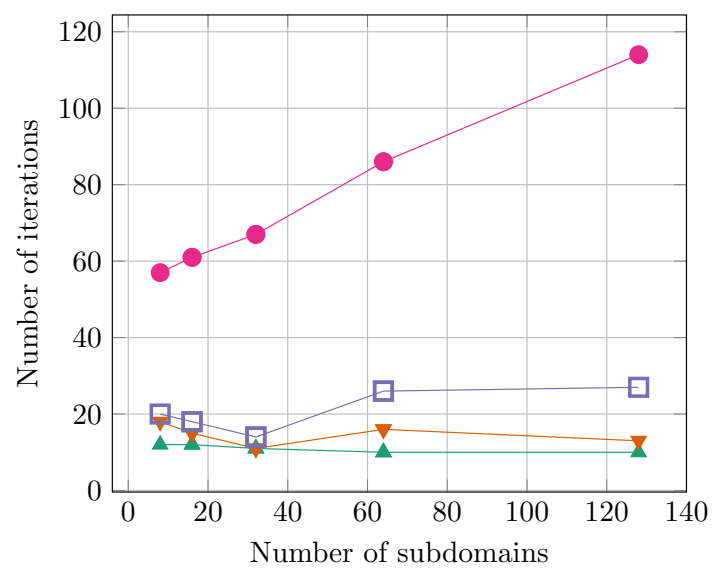

Strong scaling in 2D with GMRes

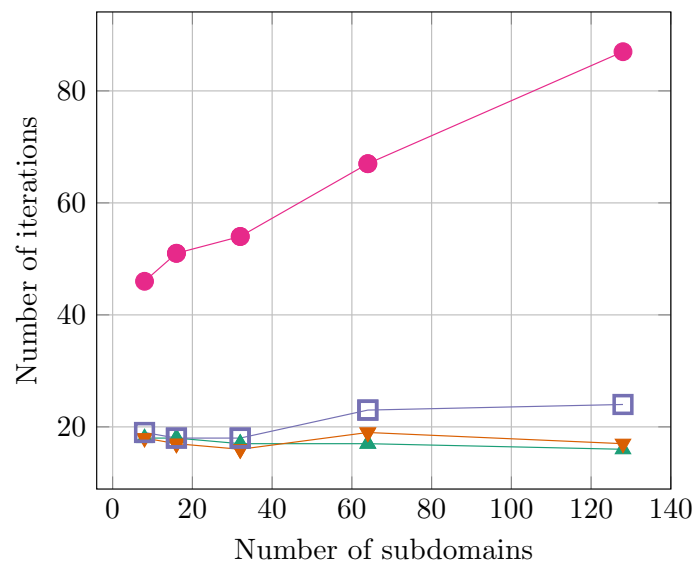

$\neg$ Stiffness $\quad \rightarrow \quad$ Slobodeckij $\quad \square \quad$ Single layer $\quad \bullet \quad$ One level

Figure 8: Strong scaling to solve Equation (21) with the geometry described in Figure 6

vary with the number of subdomains. It is interesting to see how the mean local contribution decreases with the number of subdomains so that the size of the global coarse space does not increase more than linearly.

Finally, we also did the experiment with a Calderón preconditioning based on the weakly singular operator $V$ defined as

$$
\langle V u, v\rangle_{H^{1 / 2}(\Gamma) \times \widetilde{H}^{-1 / 2}(\Gamma)}=\int_{\Gamma \times \Gamma} G(\mathbf{x}, \mathbf{y}) u(\mathbf{x}) v(\mathbf{y}) \mathrm{d} \gamma(\mathbf{x}, \mathbf{y}),
$$

that we discretize like the hypersingular operator (see Section 1.3) using $\widetilde{\mathcal{S}}_{h}^{l}\left(\mathcal{T}\right.$ ), we denote $\mathbf{V}_{h}$ the resulting matrix. It is also symmetric positive definite according to [28, Theorem 3.5.4.]. In this case, the preconditioner is defined as [30, (5.15)]

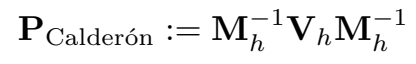

where $\mathbf{M}_{h}$ is the global mass matrix. Using $\mathbf{P}_{\text {Calderón }}$ to precondition the linear system associated with the considered test case, we obtain 17 iterations with CG and GMRes.

To conclude, we were able to obtain a number of iterations comparable to Calderón preconditioning with the GenEO stiffness coarse space and GenEO single layer coarse space, while maintaining a good behavior when increasing the number of subdomains. In this case, DDM preconditioners are interesting alternatives to Calderón preconditioning because they are less expensive to compute since only local computations are needed while Calderón preconditioning requires a global mass matrix, its inversion and a matrix-vector product with another BEM matrix. They are also more likely to scale better thanks to their parallel nature, and they give similar performances according to our results.

\subsection{D weak scaling}

We consider the following array of mesh sizes: $h=[0.0005,0.001,0.002,0.004,0.008]$ that will be used to discretize the geometry shown in Figure 6. For each mesh size $h[i]$, we obtain a 
Strong scaling in 2D

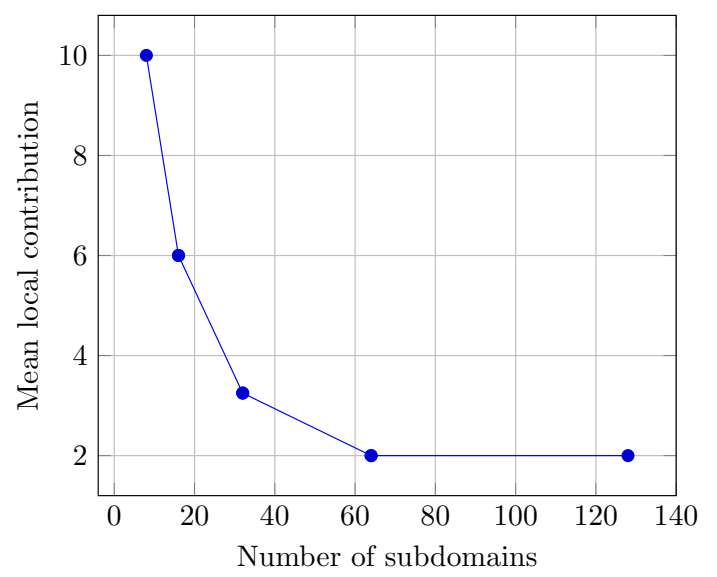

Strong scaling in $2 \mathrm{D}$

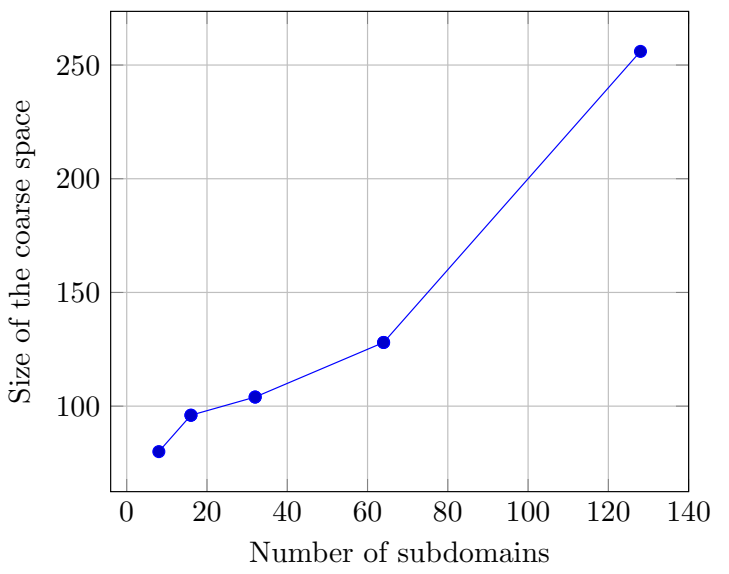

Figure 9: Mean local contribution to coarse space and size of the latter for 2D strong scaling

number of degrees of freedom $N[i]$ with $N=[48000,24000,12000,6000,3000]$. We associate a number of subdomains $n[i]$ to each mesh such that $N[i] / n[i]=750$. The resulting array of number of subdomains is $n=[64,32,16,8,4]$. The results about the number of iterations are given Figure 10. The same remarks as for the strong scaling can be formulated here.

\subsection{D test case}

In agreement with the fact that Theorems 2 to 4 do not depend on the geometric dimension, we observe similar results to the $2 \mathrm{D}$ case. We consider a similar test case, with cubes instead of squares. To be more precise, we have now $\Omega_{\text {out }}=[-2,2] \times[-2,2] \times[-2,2] \subset \mathbb{R}^{3}$ and $\Omega_{\text {in }}=[-1,1] \times[-1,1] \times[-1,1] \subset \mathbb{R}^{3}$ as described in Figure 7 , but we still consider Equation $(21)$ with the same boundary condition. The main difference, besides the geometry, is the Green function which is now defined as $G(\mathbf{z}):=\frac{1}{4 \pi} \frac{e^{-\kappa\|\mathbf{z}\|}}{\|\mathbf{z}\|}$.

\subsection{D strong scaling}

We take a mesh size of 0.039 so that we obtain a number of degrees of freedom $N$ equal to 104738. We solve the linear system associated with our test case and preconditioned with the coarse spaces we introduced earlier using several numbers of subdomains: $\{32,64,128,256,512\}$. We only add one layer of elements for the overlap. As for the $2 \mathrm{D}$ strong scaling, we take a fixed $\tau$, here equal to 5 , for the GenEO stiffness coarse space, and we take the same number of eigenvectors in the local eigenproblems to build the coarse component of the other coarse spaces.

The resulting numbers of iterations are shown in Figure 12. We observe the same behavior as in 2D. Note that here the number of iterations without preconditioner is 156 with CG and 101 with GMRes.

\subsection{D weak scaling}

We consider the following array of mesh sizes: $h=[0.05,0.07,0.1,0.15,0.2]$. For each mesh size $h[i]$, we obtain a number of degrees of freedom $N[i]$ with $N=[63360,33200,15922,7201,3846]$. We associate a number of subdomains $n[i]$ to each mesh such that $N[i] / n[i] \simeq 1000$. The resulting 

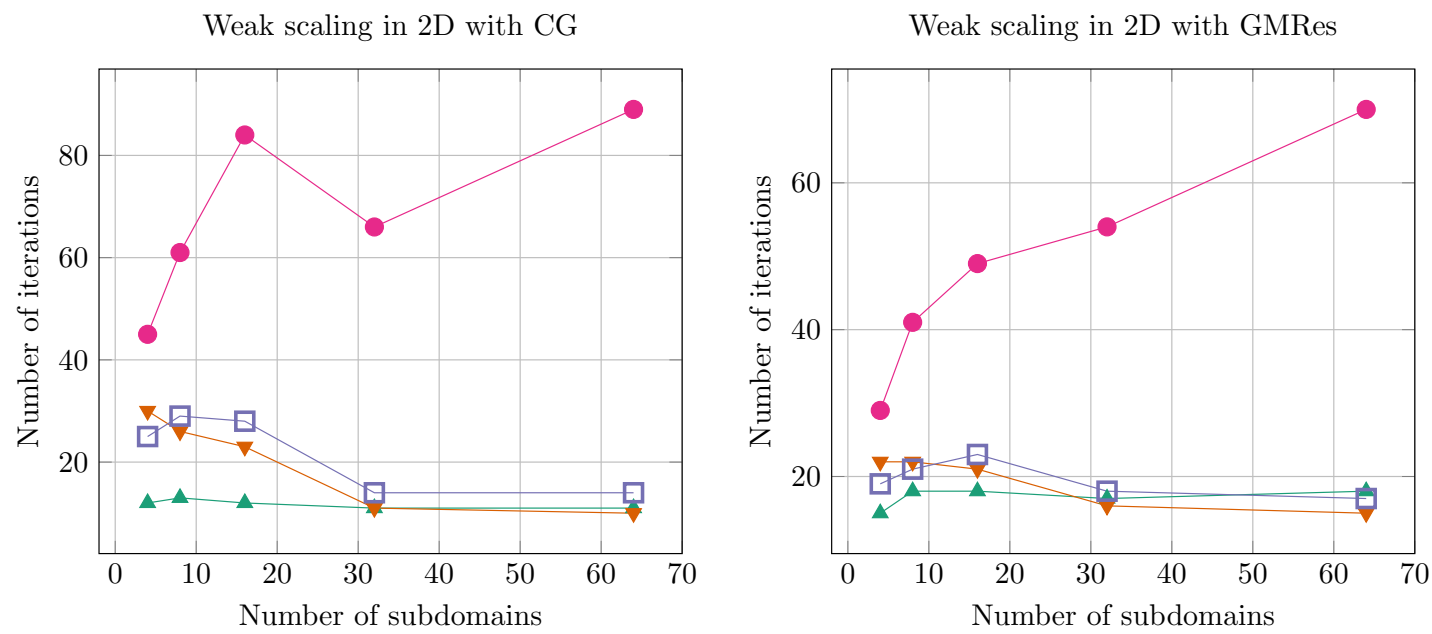

$$
\neg \text { Stiffness } \multimap-\quad \text { Slobodeckij } \quad \square-\text { Single layer } \quad \bullet \quad \text { One level }
$$

Figure 10: Weak scaling to solve Equation (21) with the geometry described in Figure 6
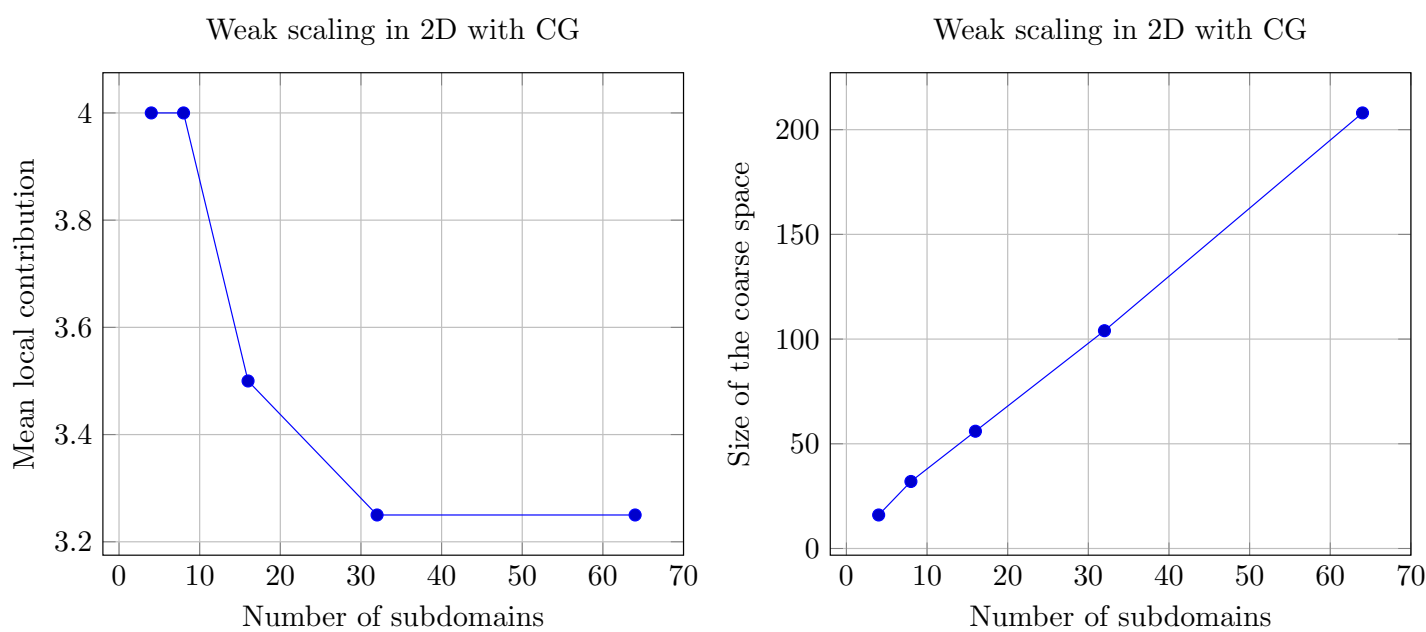

Figure 11: Mean local contribution to coarse space and size of the latter for $2 \mathrm{D}$ weak scaling 
Strong scaling in 3D with CG

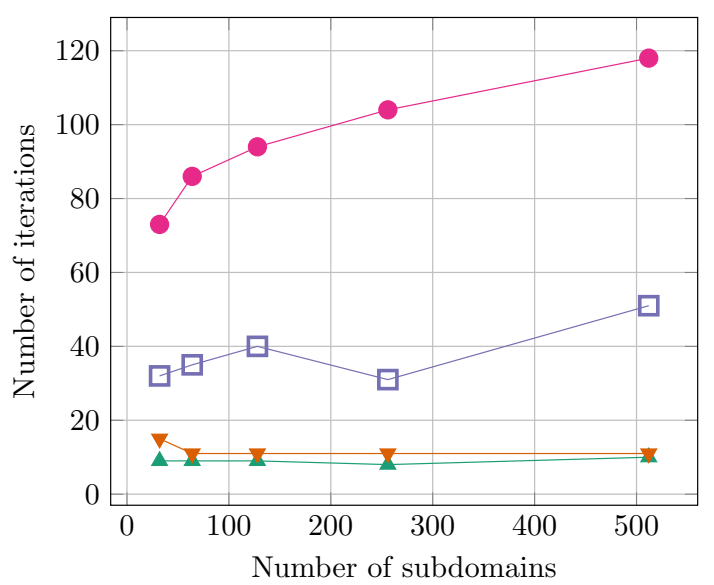

Strong scaling in 3D with GMRes

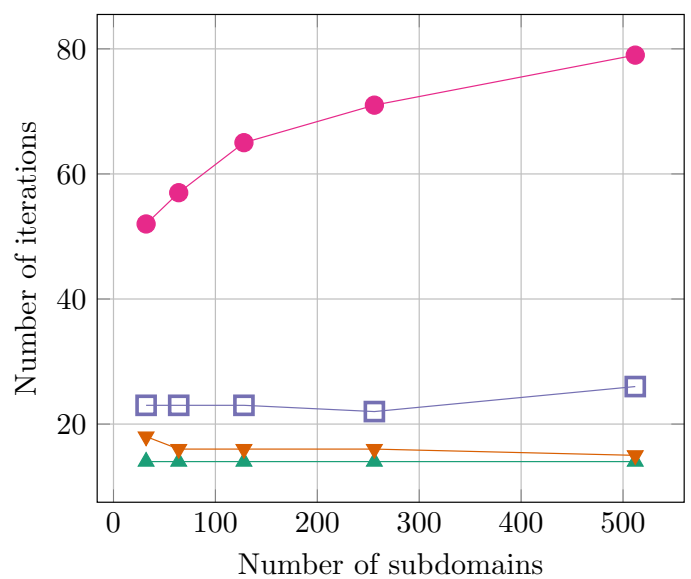

$\neg$ Stiffness $\rightarrow$ Slobodeckij $\quad \square \quad$ Single layer $\quad-\quad$ One level

Figure 12: Strong scaling to solve Equation (21) in 3D

Strong scaling in 3D

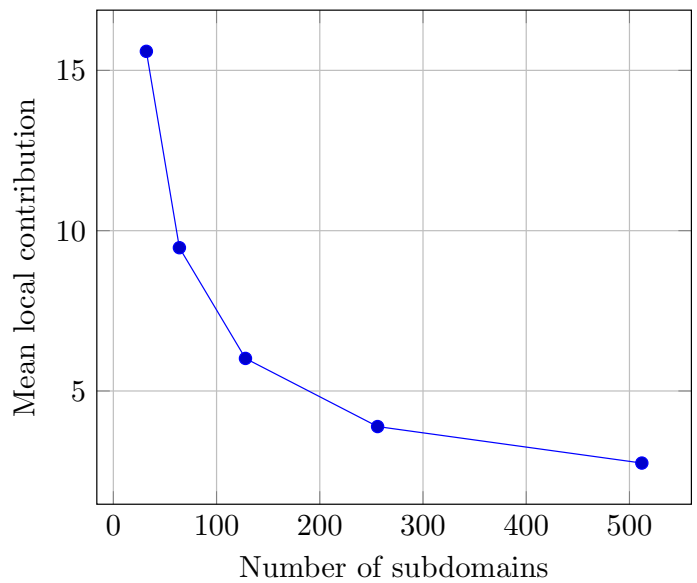

Strong scaling in 3D

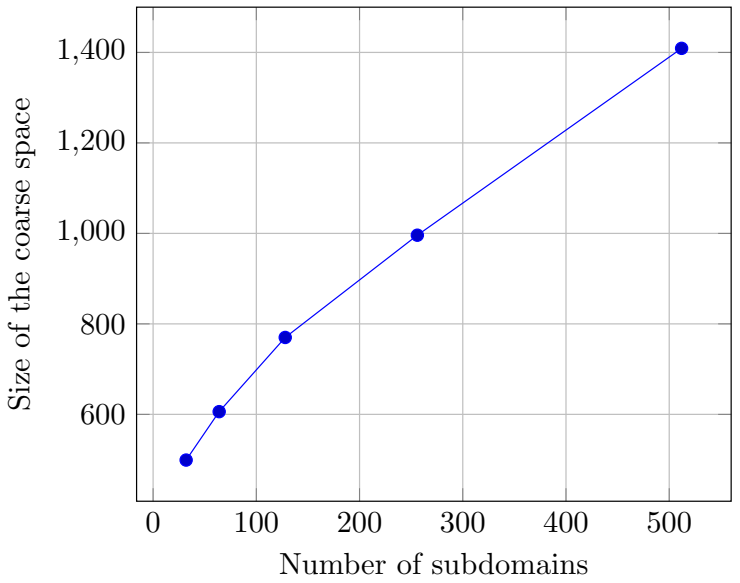

Figure 13: Mean local contribution to coarse space and size of the latter for 3D strong scaling 
Weak scaling in 3D with $\mathrm{CG}$

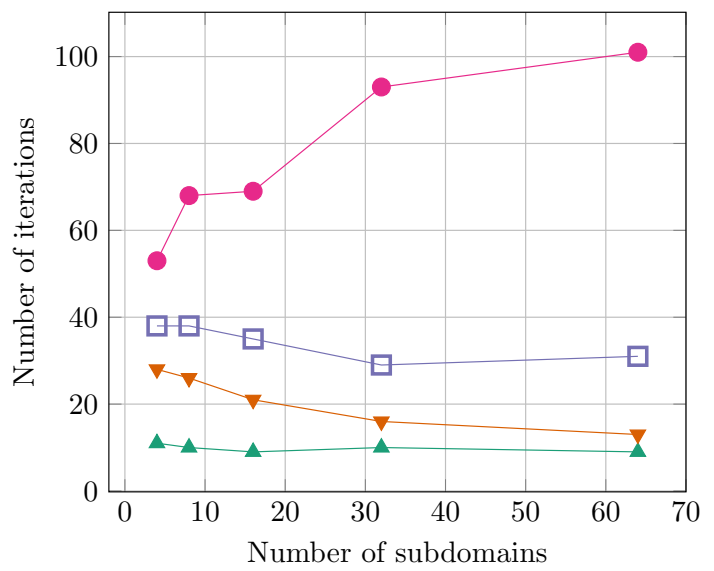

Weak scaling in 3D with GMRes

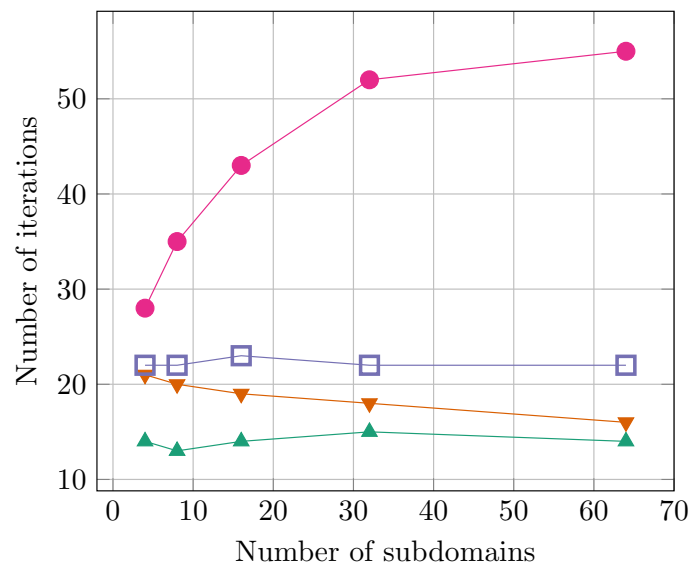

$\neg$ Stiffness $\rightarrow-$ Slobodeckij $\quad \square \quad$ Single layer $\quad-\quad$ One level

Figure 14: Weak scaling to solve Equation (21) in 3D

array of number of subdomains is $n=[64,32,16,8,4]$. The results about the number of iterations are given in Figure 14. The same remarks as for the strong scaling can be made here. But note that the $3 \mathrm{D}$ weak scaling is approximated contrary to the $2 \mathrm{D}$ weak scaling.

\section{Acknowledgments}

This work received support from the ANR research grant ANR-15-CE23-0017-01 and was granted access to the HPC resources of CINES under the allocation 2018-A0060607330 made by GENCI.

\section{References}

[1] Ainsworth, M., And Guo, B. An additive Schwarz preconditioner for p-version boundary element approximation of the hypersingular operator in three dimensions. Numerische Mathematik 85, 3 (2000), 343-366.

[2] Alléon, G., Benzi, M., And Giraud, L. Sparse approximate inverse preconditioning for dense linear systems arising in computational electromagnetics. Numerical Algorithms 16, 1 (1997), 1-15.

[3] Alouges, F., And Aussal, M. The sparse cardinal sine decomposition and its application for fast numerical convolution. Numerical Algorithms 70, 2 (jan 2015), 427-448.

[4] Anderson, E., Bai, Z., Bischof, C., Blackford, S., Demmel, J., Dongarra, J., Du Croz, J., Greenbaum, A., Hammerling, S., McKenney, A., And Sorensen, D. LAPACK Users' Guide, vol. 9. SIAM, 1999.

[5] Aurada, M., Feischl, M., Führer, T., Karkulik, M., and Praetorius, D. Energy norm based error estimators for adaptive BEM for hypersingular integral equations. Applied Numerical Mathematics 95 (sep 2015), 15-35. 
Weak scaling in 3D with CG

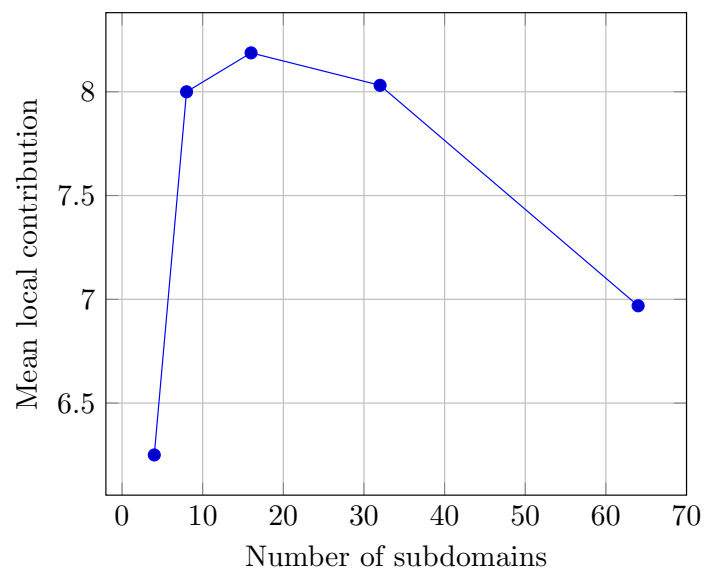

Weak scaling in 3D with CG

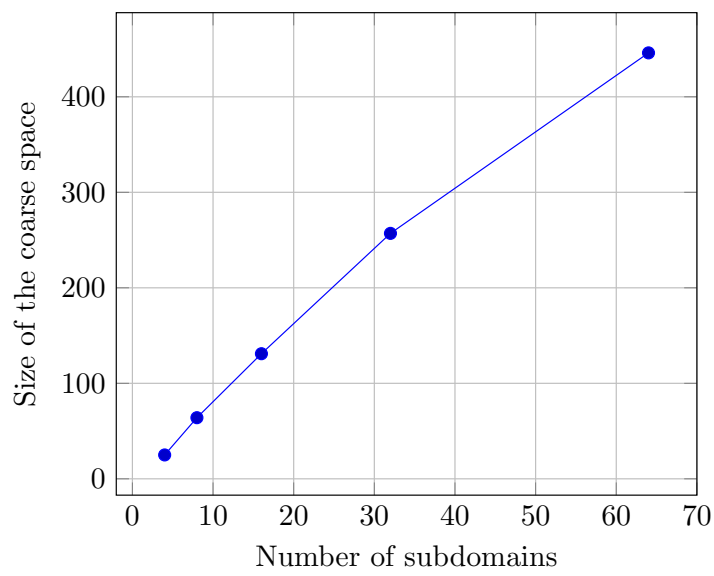

Figure 15: Mean local contribution to coarse space and size of the latter for 3D weak scaling

[6] Bebendorf, M. Hierarchical matrices: A Means to Efficiently Solve Elliptic Boundary Value Problems, vol. 63 of Lecture Notes in Computational Science and Engineering. Springer-Verlag, Berlin, 2008.

[7] NIST Digital Library of Mathematical Functions. http://dlmf.nist.gov/, Release 1.0.21 of 2018-12-15. F. W. J. Olver, A. B. Olde Daalhuis, D. W. Lozier, B. I. Schneider, R. F. Boisvert, C. W. Clark, B. R. Miller and B. V. Saunders, eds.

[8] Dolean, V., Jolivet, P., And Nataf, F. An introduction to domain decomposition methods. Society for Industrial and Applied Mathematics (SIAM), Philadelphia, PA, 2015. Algorithms, theory, and parallel implementation.

[9] Gander, M. J. Schwarz methods over the course of time. Electronic Transactions on Numerical Analysis 31 (2008), 228-255.

[10] Geuzaine, C., And Remacle, J.-F. Gmsh: a 3-D finite element mesh generator with built-in pre- and post-processing facilities. International Journal for Numerical Methods in Engineering 79, 11 (2009), 1309-1331.

[11] Greengard, L., Gueyffier, D., Martinsson, P.-G., and Rokhlin, V. Fast direct solvers for integral equations in complex three-dimensional domains. Acta Numerica 18 (may 2009), 243.

[12] Greengard, L., And Rokhlin, V. A fast algorithm for particle simulations. Journal of Computational Physics 73, 2 (dec 1987), 325-348.

[13] Griebel, M., AND Oswald, P. On the abstract theory of additive and multiplicative Schwarz algorithms. Numerische Mathematik 70, 2 (1995), 163-180.

[14] Hackbusch, W. Hierarchical Matrices: Algorithms and Analysis, vol. 49 of Springer Series in Computational Mathematics. Springer-Verlag, Berlin, 2016.

[15] Hestenes, M., And Stiefel, E. Methods of conjugate gradients for solving linear systems. Journal of Research of the National Bureau of Standards 49, 6 (1952), 409-436. 
[16] Heuer, N. Additive Schwarz Methods for Weakly Singular Integral Equations In $\mathbb{R}^{3}$ - the p-Version. Vieweg+Teubner Verlag, Wiesbaden, 1996, pp. 126-135.

[17] Hiptmair, R. Operator preconditioning. Computers \& Mathematics with Applications 52, 5 (2006), 699-706.

[18] Hiptmair, R., Jerez-Hanckes, C., and Urzúa-Torres, C. Optimal operator preconditioning for hypersingular operator over 3d screens. Tech. Rep. 2016-09, Seminar for Applied Mathematics, ETH Zürich, Switzerland, 2016.

[19] Jolivet, P., Hecht, F., Nataf, F., and Prud'homme, C. Scalable domain decomposition preconditioners for heterogeneous elliptic problems. In Proceedings of the International Conference for High Performance Computing, Networking, Storage and Analysis on - SC '13 (2013), ACM Press.

[20] Karypis, G., And Kumar, V. A fast and high quality multilevel scheme for partitioning irregular graphs. SIAM Journal on Scientific Computing 20, 1 (1998), 359-392.

[21] Martinsson, P., And Rokhlin, V. A fast direct solver for boundary integral equations in two dimensions. Journal of Computational Physics 205, 1 (may 2005), 1-23.

[22] McLean, W. Strongly Elliptic Systems and Boundary Integral Equations. Cambridge University Press, Cambridge, 2000.

[23] McLean, W., and Steinbach, O. Boundary element preconditioners for a hypersingular integral equation on an interval. Advances in Computational Mathematics 11, 4 (Dec 1999), $271-286$.

[24] Nepomnyaschikh, S. V. Decomposition and fictitious domains methods for elliptic boundary value problems. In Fifth International Symposium on Domain Decomposition Methods for Partial Differential Equations (1992), Philadelphia, PA: Society for Industrial and Applied Mathematics, pp. 62-72.

[25] RJasanow, S., and Steinbach, O. The Fast Solution of Boundary Integral Equations. Mathematical and Analytical Techniques with Applications to Engineering. Springer Science \& Business Media, 2007.

[26] SAAd, Y. Iterative Methods for Sparse Linear Systems. Society for Industrial and Applied Mathematics, jan 2003.

[27] SAAD, Y., AND Schultz, M. H. GMRES: a generalized minimal residual algorithm for solving nonsymmetric linear systems. SIAM Journal on Scientific and Statistical Computing 7, 3 (1986), 856-869.

[28] Sauter, S. A., and Schwab, C. Boundary element methods, vol. 39 of Springer Series in Computational Mathematics. Springer-Verlag, Berlin, 2011. Translated and expanded from the 2004 German original.

[29] Spillane, N., Dolean, V., Hauret, P., Nataf, F., Pechstein, C., and Scheichl, R. Abstract robust coarse spaces for systems of PDEs via generalized eigenproblems in the overlaps. Numerische Mathematik 126, 4 (2014), 741-770.

[30] Steinbach, O., and Wendland, W. L. The construction of some efficient preconditioners in the boundary element method. Advances in Computational Mathematics 9, 1-2 (1998), 191-216. Numerical treatment of boundary integral equations. 
[31] Stephan, E. P. Boundary integral equations for screen problems in $\mathbb{R}^{3}$. Integral Equations and Operator Theory 10, 2 (1987), 236-257.

[32] Toselli, A., And Widlund, O. B. Domain decomposition methods: algorithms and theory, vol. 34. Springer, 2005.

[33] Tran, T., And Stephan, E. P. Additive Schwarz methods for the h-version boundary element method. Applicable Analysis 60, 1-2 (1996), 63-84.

[34] West, D. B. Introduction to Graph Theory (2nd Edition). Pearson, 2000.

[35] Widlund, O., And Dryja, M. An additive variant of the Schwarz alternating method for the case of many subregions. Tech. Rep 339, Department of Computer Science, Courant Institute, 1987. 